\title{
L'élevage des yaks au Bhoutan : I. Diversité des structures d'élevage et des fonctionnements technico-économiques : quels indicateurs systémiques de viabilité ?
}

Yak husbandry in Bouthan: I. Diversity of herding patterns and technicaleconomic procedures : what are the systemic indicators of viability?

Marie Dervillé

\section{(2) OpenEdition}

Édition électronique

URL : https://journals.openedition.org/emscat/2149

DOI : $10.4000 /$ emscat.2149

ISSN : 2101-0013

Éditeur

Centre d'Etudes Mongoles \& Sibériennes / École Pratique des Hautes Études

Référence électronique

Marie Dervillé, «L'élevage des yaks au Bhoutan : I. Diversité des structures d'élevage et des fonctionnements technico-économiques : quels indicateurs systémiques de viabilité ? ", Études mongoles et sibériennes, centrasiatiques et tibétaines [En ligne], 43-44 | 2013, mis en ligne le 20 septembre 2013, consulté le 13 juillet 2021. URL : http://journals.openedition.org/emscat/2149; DOI : https://doi.org/10.4000/emscat.2149

Ce document a été généré automatiquement le 13 juillet 2021

(ㄷ) Tous droits réservés 


\section{L'élevage des yaks au Bhoutan : I. Diversité des structures d'élevage et des fonctionnements technico- économiques : quels indicateurs systémiques de viabilité ?}

Yak husbandry in Bouthan: I. Diversity of herding patterns and technicaleconomic procedures : what are the systemic indicators of viability?

Marie Dervillé

\section{Introduction}

1 Le Bhoutan est un petit pays de $38394 \mathrm{~km}^{2}$ pour 672425 habitants (Royal Government of Bhutan 2005) coincé entre deux géants, l'Inde et la Chine. Le Bhoutan recouvre une diversité d'écosystèmes, des plaines tropicales humides de la frontière indienne au climat alpin des sommets himalayens en bordure du plateau tibétain. Sur $150 \mathrm{~km}$, l'altitude passe de 150 à $7300 \mathrm{~m}$. La géographie du pays est d'autant plus complexe que les vallées orientées nord/sud sont séparées de cols à près de $4000 \mathrm{~m}$. La principale zone de peuplement est la zone centrale comprise entre 2000 et $3500 \mathrm{~m}$ d'altitude. Elle est caractérisée par un climat alpin et une saison des pluies peu marquée. La polyculture-élevage y est traditionnellement pratiquée. D'ouest en est la culture du blé, du riz, du sarrasin et du maïs dominent successivement. Au-delà de $3000 \mathrm{~m}$, le nord du Bhoutan au climat rigoureux est peuplé d'éleveurs de yaks. Le développement du sud du pays est plus récent (Pommaret 2000). Les plantations d'orangers et de cardamome y jouent un rôle prépondérant depuis le défrichement engagé au début du $\mathrm{xx}^{\mathrm{e}}$ siècle. 
Figure 1 : Carte administrative du Bhoutan : 20 régions (dzongkhag)

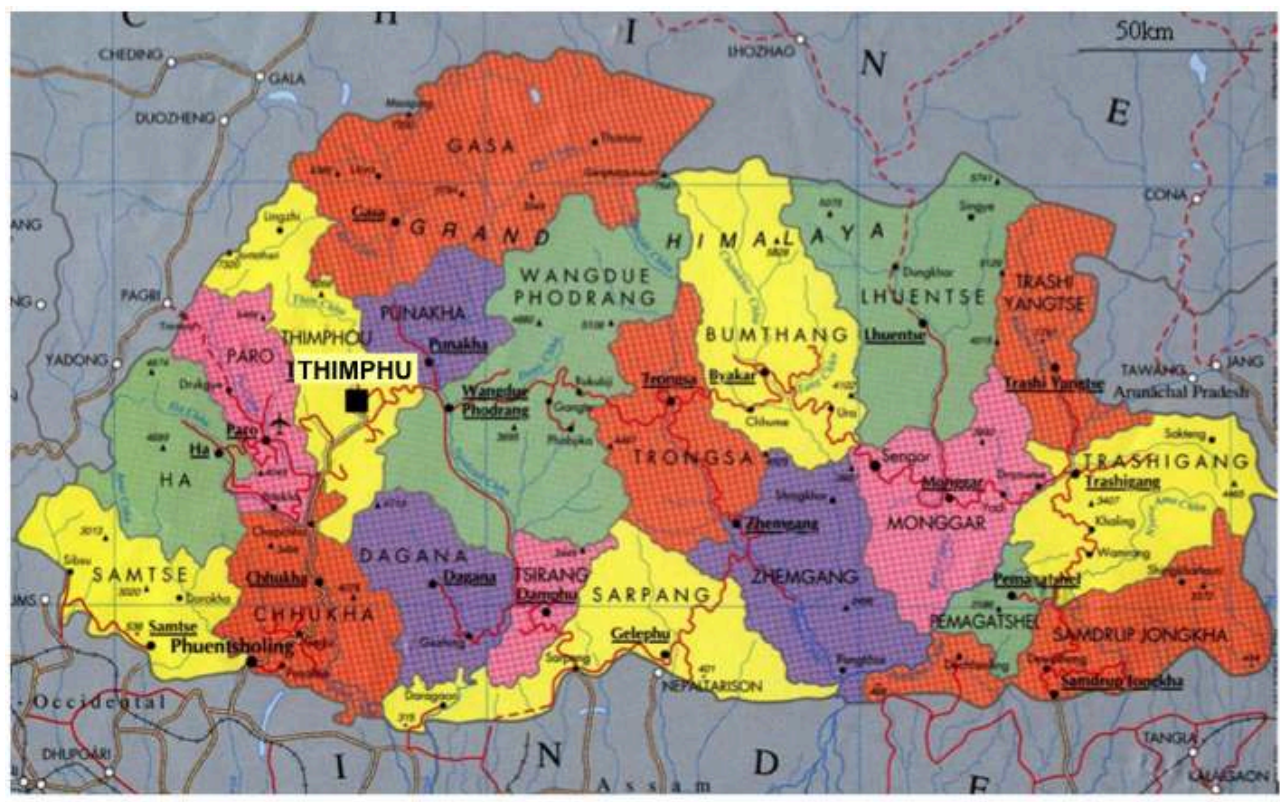

Le Bhoutan est administrativement divisé en 20 régions, elles-mêmes divisées en geogs ${ }^{1}$ (fig. 1). L'élevage de yaks concerne la partie septentrionale d'une dizaine de régions : Paro, Haa, Thimphu, Gaza, Wangdue-Phodrang, Trongsa, Bumthang, Lhuentse, TrashiYangtse et Trashigang. Les éleveurs de yaks au Bhoutan sont semi-nomades ${ }^{2}$, ils se déplacent avec leurs animaux des pâturages d'hiver de basse altitude (2 500-3 $000 \mathrm{~m}$ ) aux pâturages d'été (4500-5 500 m). Le village en pierre correspond au campement d'hiver, situé, selon les zones, à quelques heures ou plusieurs jours de marche de la route la plus proche (photo 1). Selon les zones, les migrations ont lieu à l'intérieur des frontières du geog ou sur plusieurs geogs. En 2008, l'activité comptait entre 1000 et 1400 foyers pour un cheptel d'environ 48400 animaux (Department of Livestock 2008)3. 
Photo 1. Village d'hiver d'éleveurs de yaks du système Centre

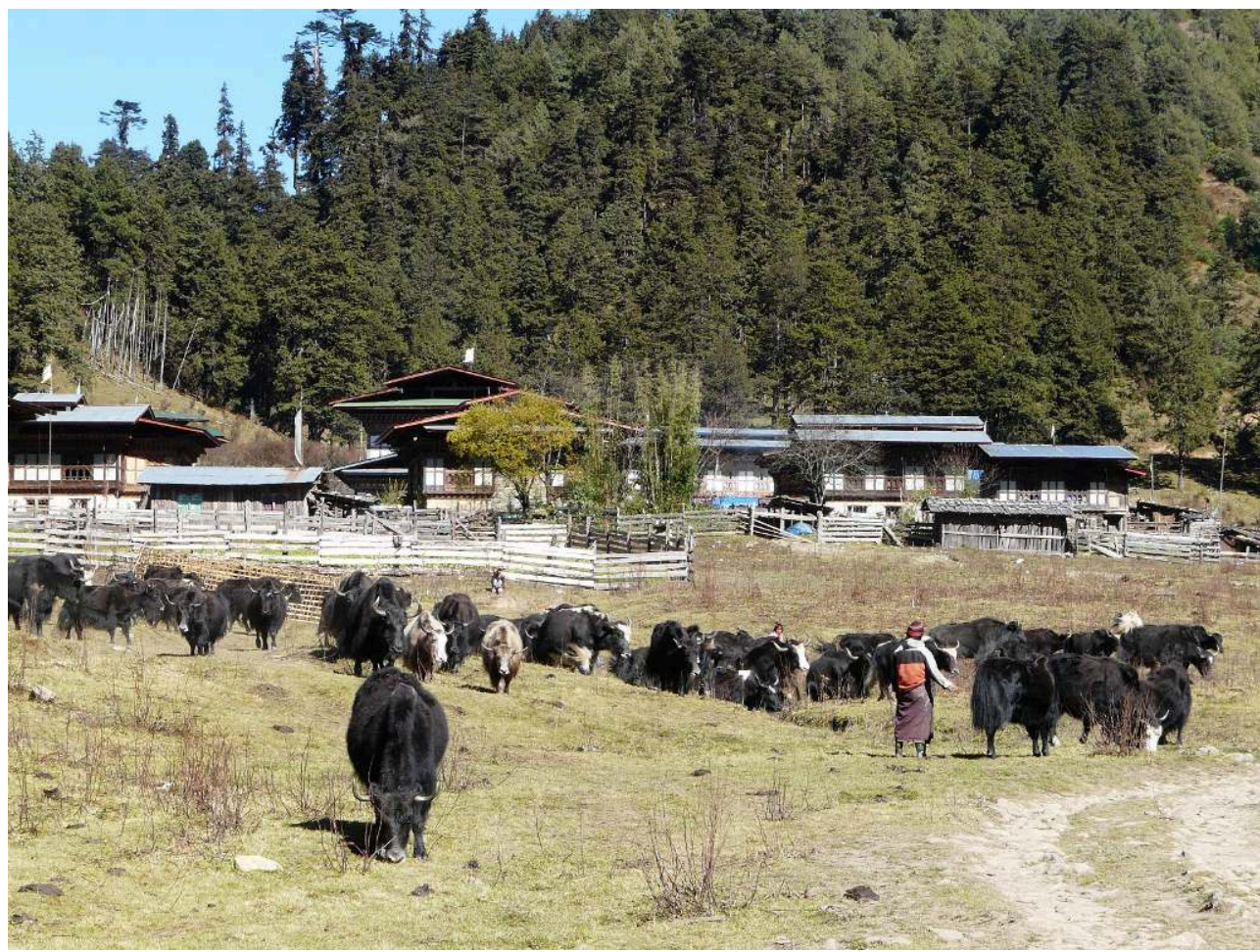

Les femelles et les jeunes animaux, après avoir passé la nuit près du village, sont envoyés pâturer pour la journée

Marie Dervillé (novembre 2009, à Ganchuka dans le geog de Sephu, dans le Dzongkhag de Wangdue, altitude approximative $3200 \mathrm{~m}$, à 45 min de marche de la route la plus proche)

3 L'élevage de yaks a fait l'objet, au Bhoutan, de travaux scientifiques dans différents domaines: économie politique (Karma Ura 1993, 2002), agronomie et systèmes fourragers (Pema Gyamthso 1996, Roder \& Pema Gyamtsho 2001), génétique (Tashi Dorji 2000). Néanmoins aucun de ces travaux n'aborde l'activité d'élevage dans sa globalité, ce qui limite les possibilités d'analyse de sa viabilité à court et moyen terme.

4 L'hypothèse formulée dans cet article est que l'analyse systémique peut permettre de caractériser les performances technico-économiques de l'élevage de yaks et d'en évaluer la viabilité.

5 Le département d'élevage bhoutanais s'est progressivement laissé convaincre de la pertinence de la mobilisation d'une telle approche. À l'aide de la littérature existante et d'entretiens auprès d'experts nationaux il a été décidé de travailler sur quatre territoires contrastés avec, d'ouest en est : le geog de Bjee dans la région de Haa, les geog de Soe et de Lingshi dans le région de Thimphu, le geog de Choekhor dans la région de Bumthang, le geog de Merak dans la région de Trashigang enfin.

\section{Problématique et démarche méthodologique}

\section{Le cadre d'analyse des systèmes d'élevage de yaks au Bhoutan}

6 Un système d'élevage est un ensemble d'éléments en interaction dynamique organisé par l'homme en vue de valoriser des ressources par l'intermédiaire d'animaux domestiques pour en obtenir des productions variées (lait, viande, cuir, peau, travail, 
fumure, etc.) ou pour répondre à d'autres objectifs (Landais 1994). L'objectif n'est pas de décrire de façon exhaustive la multiplicité des pratiques d'élevage et leurs performances technico-économiques mais de construire une représentation finalisée du réel (Landais 1994) ; une représentation qui dans le cas présent permette d'apporter des éléments de réponse à la question de la capacité d'adaptation au changement de contexte économique et politique de ces élevages dans leur diversité. Le système d'élevage (Bonnemaire 2001) est un système de relations avec trois pôles - les hommes, les animaux et les ressources - qui ne peut être construit, spécifié, contextualité et modélisé qu'en fonction de projets de connaissance et/ou projets d'action (action des opérateurs ou action des chercheurs). Ce système de relations peut être décliné à différents niveaux d'organisation: exploitations, région, aire de population animale, territoire ou bassin de production. L'étude de systèmes d'élevage ainsi définis implique l'interdisciplinarité et passe par l'élaboration d'objets scientifiques hybrides (sciences biotechniques et sociales) qui peuvent être informés par la combinaison de points de vue. Chaque point de vue révèle des faits, mobilise des méthodes et des instruments particuliers, propose des logiques aux phénomènes observés ou attendus, donc identifie et définit aussi un ensemble particulier de " performances » du système.

7 L'analyse des pratiques constitue une entrée privilégiée pour l'étude des systèmes d'élevage (Landais 1994). Cet auteur propose de scinder l'analyse des pratiques d'élevage en en distinguant cinq types: agrégation, conduite (reproduction, alimentation, santé), exploitation (traite, tonte, abattage), renouvellement et valorisation (transformation et mise en marché).

Le choix a été fait d'un découpage de l'activité « élevage de yaks " par grandes zones facilitant la prise en compte de la différenciation géographique et historique des pratiques. Ordonner ainsi l'univers "élevage de yaks au Bhoutan » en types de systèmes régionaux permet de structurer l'analyse et de produire des connaissances à visée opératoire facilitant l'intervention éventuelle des acteurs du développement.

9 La viabilité est « le caractère de ce qui est viable, peut vivre, se développer ; de ce qui présente les conditions nécessaires pour durer» (Le Petit Robert 2006). La viabilité d'une activité dépend d'abord du niveau moyen de revenu qui est lui-même fonction des revenus de la production et, dans une perspective élargie, des revenus liés aux activités non-agricoles des ménages (Landais 1998).

\section{Une démarche de terrain et des questions pour comprendre et quantifier}

Dans l'étude entreprise, le dialogue avec les acteurs et l'observation de terrain ont constitué la principale source d'information. Si certains élevages (campements d'hiver) des régions de Haa et du Centre sont accessibles par route, la majorité des entretiens ont été réalisés à plusieurs jours de marche de la route la plus proche. L'éloignement des campements d'hiver est maximum dans l'Ouest du pays (quatre à cinq jours de marche de la route). Nombre d'entretiens auprès d'éleveurs du Centre du pays ont également été réalisés sur le chemin des pâturages à plusieurs jours de marche de la route. Les enquêtes ont été effectuées en logeant chez les éleveurs, ce qui m'a permis d'observer directement les pratiques : traite, abattage, transformation des produits. Les enquêtes ont eu lieu au printemps (2007) et à l'automne (2009) pour des raisons pratiques d'accessibilité des élevages. Pour compléter les déclarations des éleveurs, les 
performances de production et de transformation ont donc pu être mesurées directement, de façon ponctuelle, et ce, en dehors de la période du pic de lactation. Ces observations et mesures, même limitées, ont cependant permis d'avoir un regard critique sur le discours des éleveurs.

Le travail de terrain articule trois types d'enquêtes conduites auprès des éleveurs :

- des entretiens semi-directifs auprès des leaders locaux ou des éleveurs jugés performants par leurs confrères ou par les techniciens d'élevage (deux à trois entretiens par zone),

- des enquêtes individuelles approfondies auprès d'éleveurs guidées par une grille détaillée de questions (89 enquêtes ont été réalisées dont 82 se sont révélées exploitables). Cette formalisation a rendu transmissible l'analyse des pratiques. Elle a permis aussi aux techniciens d'élevage bhoutanais de participer à ce travail d'enquête, de multiplier ensuite les études de cas, de prendre ainsi conscience de la diversité des situations individuelles et, enfin, de confronter ces résultats à leurs représentations,

- des entretiens collectifs (un à deux de cinq à dix éleveurs par grande région) visant à discuter des contraintes et difficultés rencontrées, des attentes et projets des communautés (Haa, Linghsi, Merak, Wangdue).

Les 89 enquêtes auprès d'éleveurs, réalisées en 2007, étaient structurées autour de trois domaines :

- description générale de l'activité de l'éleveur (taille du foyer, nombre d'actifs, historique de l'activité d'élevage, accès à la terre et aux droits de pâturage, source complémentaire de revenu),

- caractérisation des pratiques d'élevage (taille du troupeau, migration, conduite, exploitation),

- description des entrées et des sorties du système (quantification des intrants, des investissements, de la main d'œuvre requise ; types, quantités et valeur des produits fabriqués, part de produits commercialisés et les marchés concernés).

L'analyse des résultats s'est traduite par la réalisation de deux types de travaux :

- une caractérisation des systèmes de production et une délimitation de types régionaux,

- une estimation des résultats technico-économiques des différents systèmes d'élevage.

À partir de la quantification des entrées et des sorties du système permise par ces 82 enquêtes approfondies, une estimation du revenu a ainsi pu être réalisée pour chacune des familles d'éleveur enquêtées.

Ce travail de formalisation de la diversité des pratiques et l'analyse statistique des performances technico-économiques des systèmes d'élevage débouchent sur une meilleure connaissance de ces systèmes dans leur globalité et de leurs tendances d'évolution. Je présenterai les résultats en deux points, qui feront l'objet des deux parties suivantes qui constituent le corps de cette contribution.

ans la deuxième partie de cet article, j'interroge donc le mode de vie des éleveurs à la fin des années 2000. Quelles sont leurs pratiques d'élevage et de transformation des produits ? Comment le travail est-il organisé au cours de l'année ? Où les produits sontils commercialisés ? Dans quels réseaux sociaux les éleveurs s'insèrent-ils?

Dans la troisième partie, les performances technico-économiques portées par ces pratiques d'exploitation sont estimées pour les différents systèmes d'élevage. À partir de la diversité régionale des pratiques et des performances, je définis quatre systèmes régionaux de production. La viabilité de l'activité d'élevage au Bhoutan peut ainsi être évaluée et discutée dans sa diversité. 


\section{L'élevage des yaks : une activité de subsistance imbriquée dans des relations sociales complexes}

Le yak (Bos grunniens) constitue une source de protéines animales (lait, le plus souvent transformé en beurre et en fromage, et viande) mais aussi de force de traction, de poils et de bourre utilisés dans la fabrication de tentes, de vêtements et de cordes. Les peaux séchées sont également utilisées comme tapis. Les bouses séchées sont utilisées comme combustible, principalement l'été lorsque éleveurs et troupeaux se trouvent au dessus de la limite des arbres. La figure 2 donne une représentation schématique de ce système d'élevage.

Figure 2. Représentation schématique du système de production traditionnel

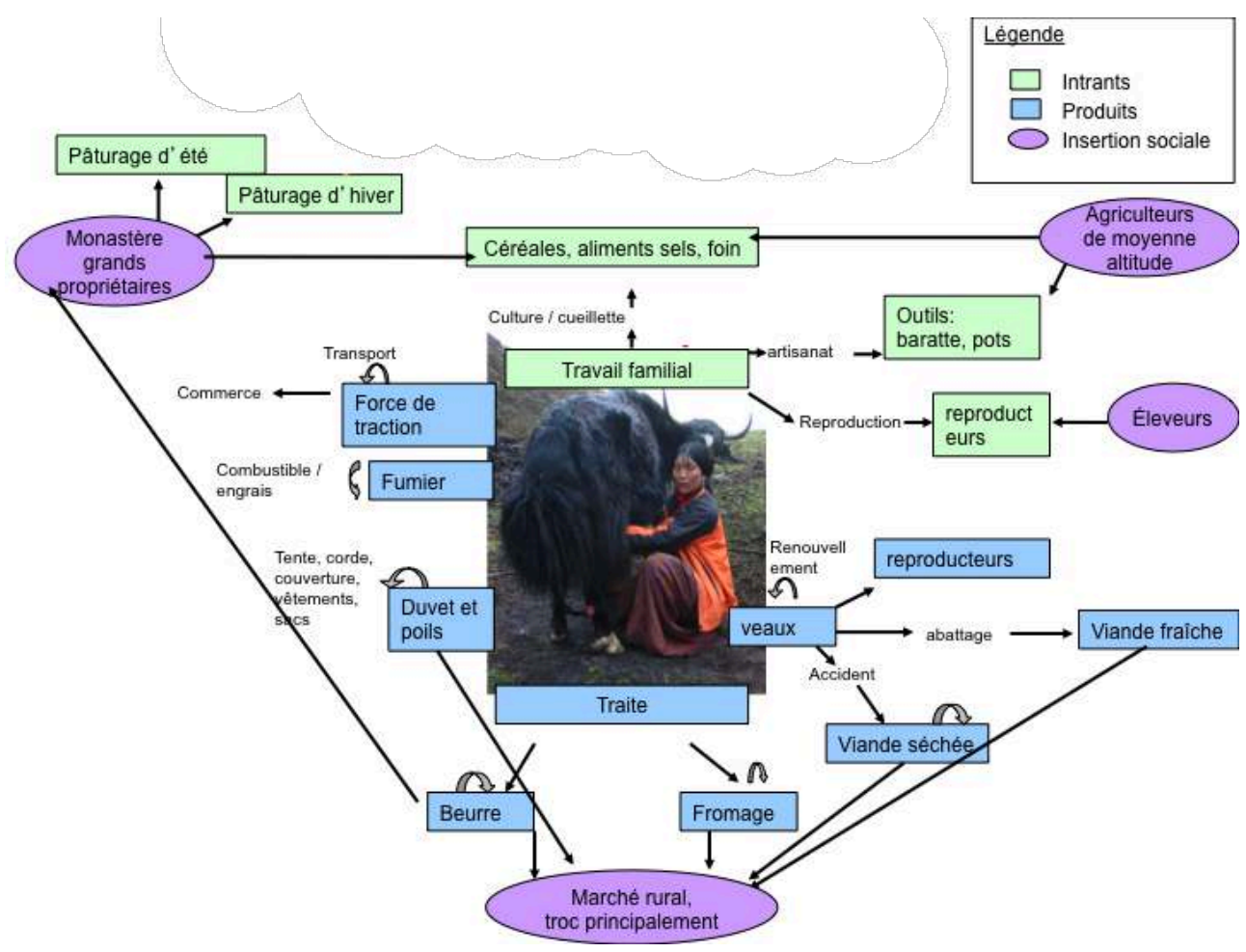

\section{Une conduite extensive du cheptel}

L'élevage de yaks est une production à base d'herbe, de prairie naturelle pâturée. Les animaux pâturent toute l'année sauf en cas de fortes chutes de neige. Chaque éleveur possède en moyenne des droits de pâturage dans huit localités différentes séparées de quelques heures à quatre à cinq jours de marche.

Le contenu de ces herbages en matière sèche et en matière nutritive varie avec la composition du sol, l'altitude, les précipitations, l'exposition et le mode de gestion. La productivité par hectare des prairies est estimée à $0,7 \mathrm{t}$ de matière sèche pour les pâturages d'hiver et à $0,4 \mathrm{t}$ pour les pâturages d'été, ce qui conduit à un besoin moyen par yak adulte de l'ordre de 3 ha en été et de 2,4 ha en hiver (Roder \& Gyamtsho 2001). 
21 Mâles et femelles sont conduits séparément. Les mâles sont envoyés sur les pâturages les plus éloignés et les plus escarpés et ils bénéficient d'une surveillance relâchée; les éleveurs ne se rendent sur place que pour rassembler les animaux en cas de besoin (migration, transport, reproduction) ou pour leur donner du sel (une fois par mois environ). Les femelles et les jeunes sont par contre rassemblés tous les soirs près du campement. Cela permet de suivre l'état sanitaire des animaux et de réaliser la traite le lendemain.

22 La complémentation de l'alimentation se limite à un apport régulier en sel (une quantité proportionnelle au poids de l'animal, en moyenne $3 \mathrm{~kg}$ par tête et par an). En hiver, les jeunes, les femelles et les animaux affaiblis bénéficient d'une ration de radis, farine, son, et plus récemment d'aliment complet acheté et d'un peu de foin les jours de neige. Selon les éleveurs, la quantité moyenne par femelle varie entre 18 et $40 \mathrm{~kg}$ de radis et 12 et $20 \mathrm{~kg}$ de farine et/ou de son par hiver. Les mâles ne reçoivent un complément de nourriture, en dehors de l'apport en sel, que les jours où ils sont utilisés pour le transport de charges. Du fait de l'éloignement, la majorité des traitements des animaux malades sont effectués par les éleveurs eux-mêmes sur la base de savoirs traditionnels (photo 2).

Photo 2. Éleveuse du système de Haa traitant un animal malade en lui crachant une concoction de plantes dans la gueule

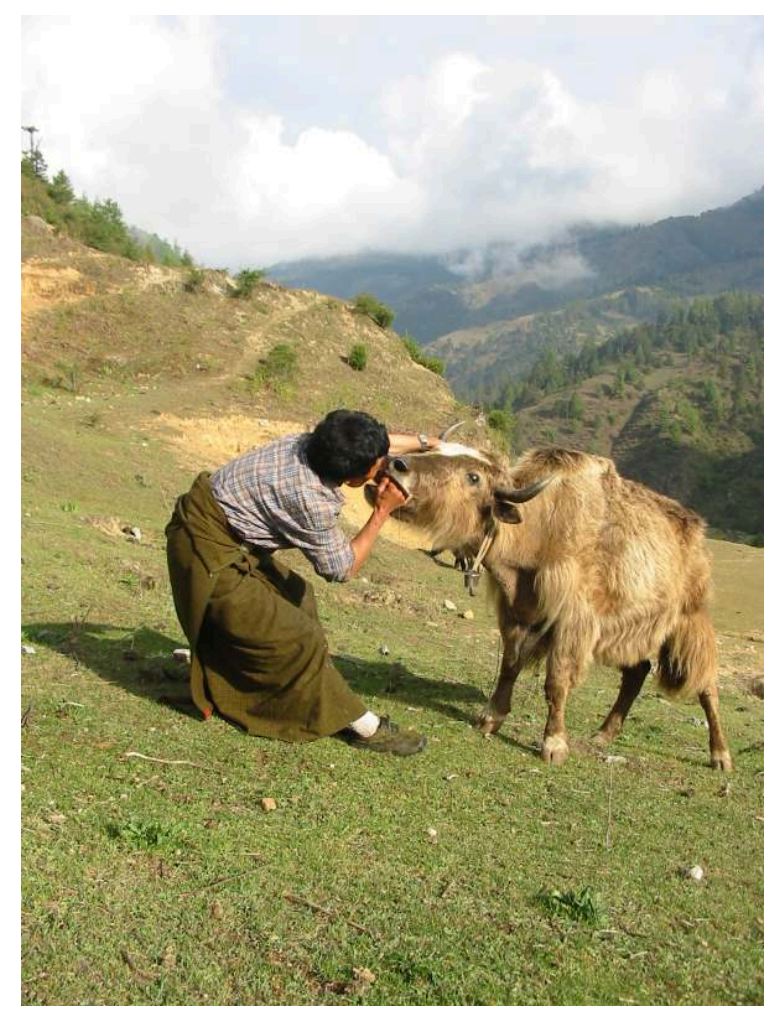

Marie Dervillé (début mai 2007, dans le geog de Bjee, dans le Dzongkhag de Haa, altitude approximative $3000 \mathrm{~m}$ )

$\mathrm{Au}$ Bhoutan, la reproduction est pratiquée essentiellement en race pure. L'hybridation avec les bovins ne concerne que les geogs de Merak et Sakteng dans la région de Trashigang, à l'est du pays ${ }^{4}$. Là, l'hybridation est pratiquée pour accroître les performances laitières des femelles (dzomo) et la force de traction des mâles $(d z o)$. Il 
s'agit d'un croisement de yaks femelles avec des taureaux d'une race tibétaine Goleng (Bos taurus) (photo 3).

Photo 3. Bos Taurus « Goleng » utilisé pour l'hybridation avec les yaks femelles (Bos Grunniens) dans le système Est

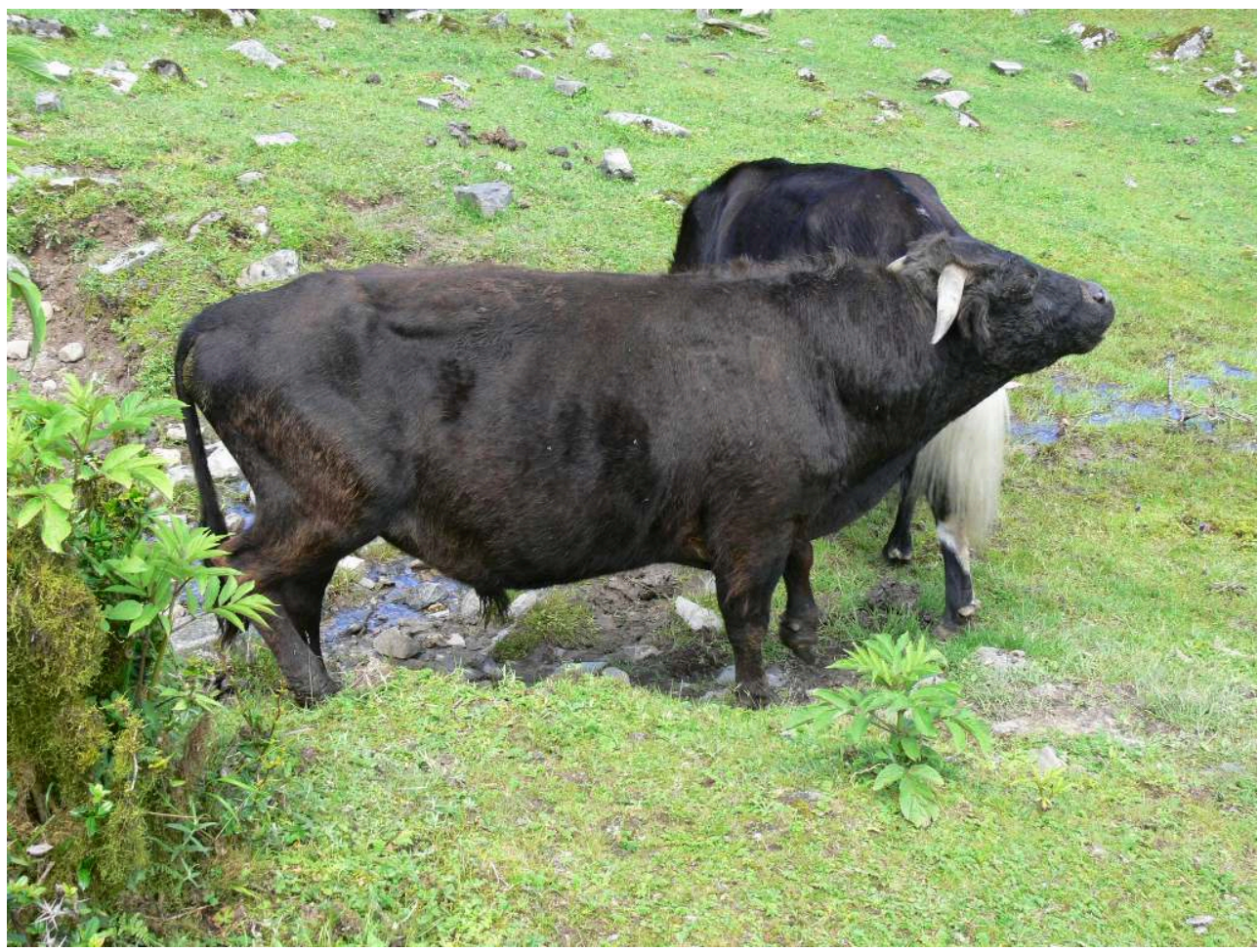

Marie Dervillé (juillet 2006, dans le geog de Sakteng, dans le Dzongkhag de Trashigang)

La reproduction des yaks est calée sur les saisons. Les accouplements ont lieu en juillet et août lorsque la température est la plus élevée, la ressource fourragère abondante, et que les animaux ont eu le temps de se remettre en condition après l'hiver. La durée de gestation étant en moyenne de 260 jours (Wiener et al. 2003), les vêlages ont lieu au printemps, de mars à juin avec un pic en mai (photo 4). Compte-tenu de pertes (cf. infra), la majorité des jeunes animaux sont gardés pour le renouvellement du troupeau. 
Photo 4. Vêlage à $5200 \mathrm{~m}$ d'altitude dans le système Ouest

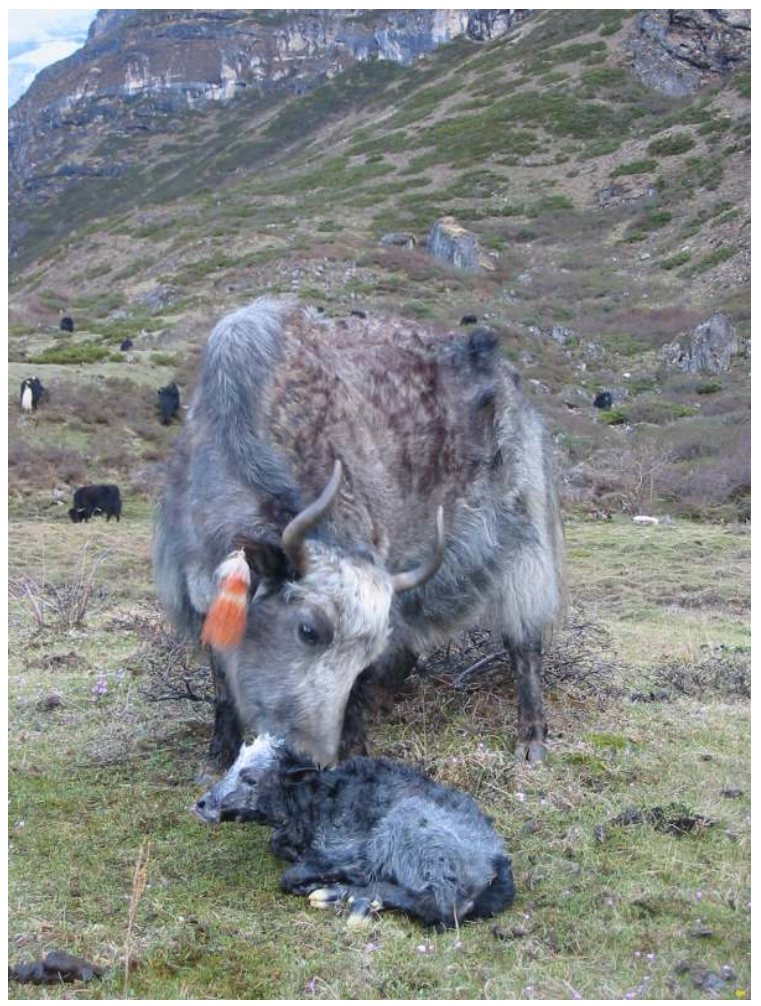

Marie Dervillé (fin mai 2007, dans le geog de Linghzi, dans le Dzongkhag de Thimphu)

25 Les femelles sont utilisées principalement pour la reproduction et la traite (photos 5 \& 6). Elles ne commencent à être vendues pour leur viande qu'entre 12 et 15 ans lorsque leur fécondité décroît. Les mâles sont utilisés soit comme reproducteur soit comme animal de bât après castration. Lorsque leurs performances diminuent à partir de 8 ans, ils commencent à être vendus sur pied pour la production de viande. Le recours à l'abattage est fréquent à l'ouest alors qu'il est peu pratiqué au centre et à l'est du pays. 
Photo 5. Traite dans le système Est

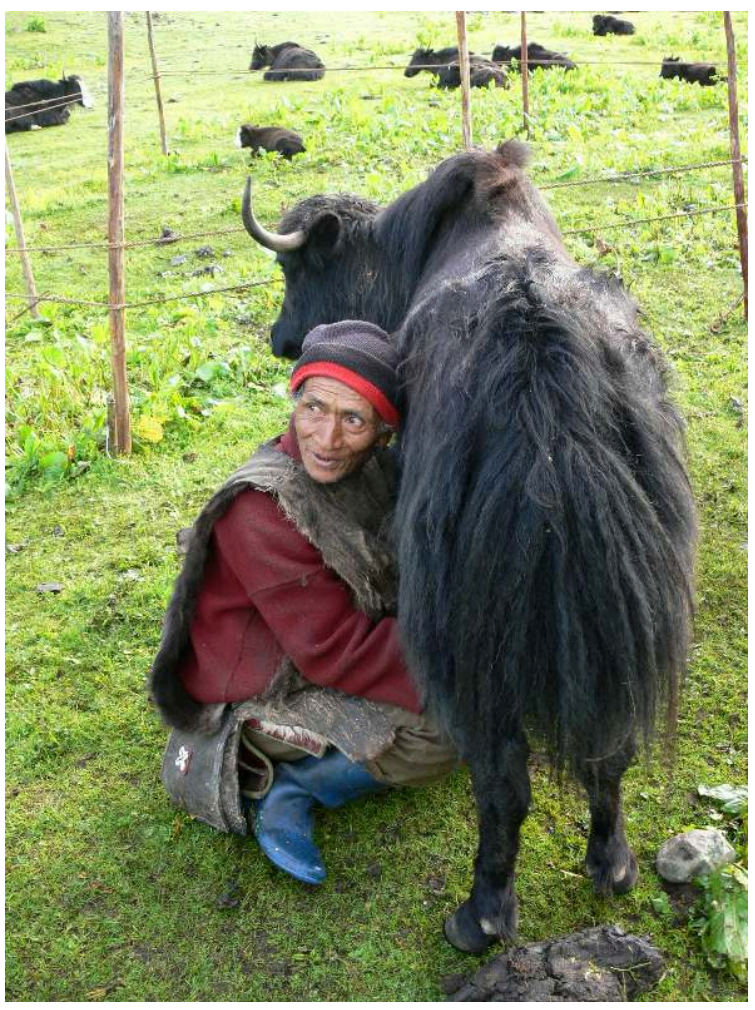

On aperçoit l'enclos dans lesquels les veaux sont attachés pendant la nuit. Les femelles restent ainsi à proximité du campement, ce qui permet aux éleveurs de gagner du temps le matin pour la traite Marie Dervillé (juin 2007, dans le geog de Merak, dans le Dzongkhag de Trashigang, altitude approximative $3400 \mathrm{~m}$ ) 
Photo 6. Femelles à l'attache dans le système Ouest

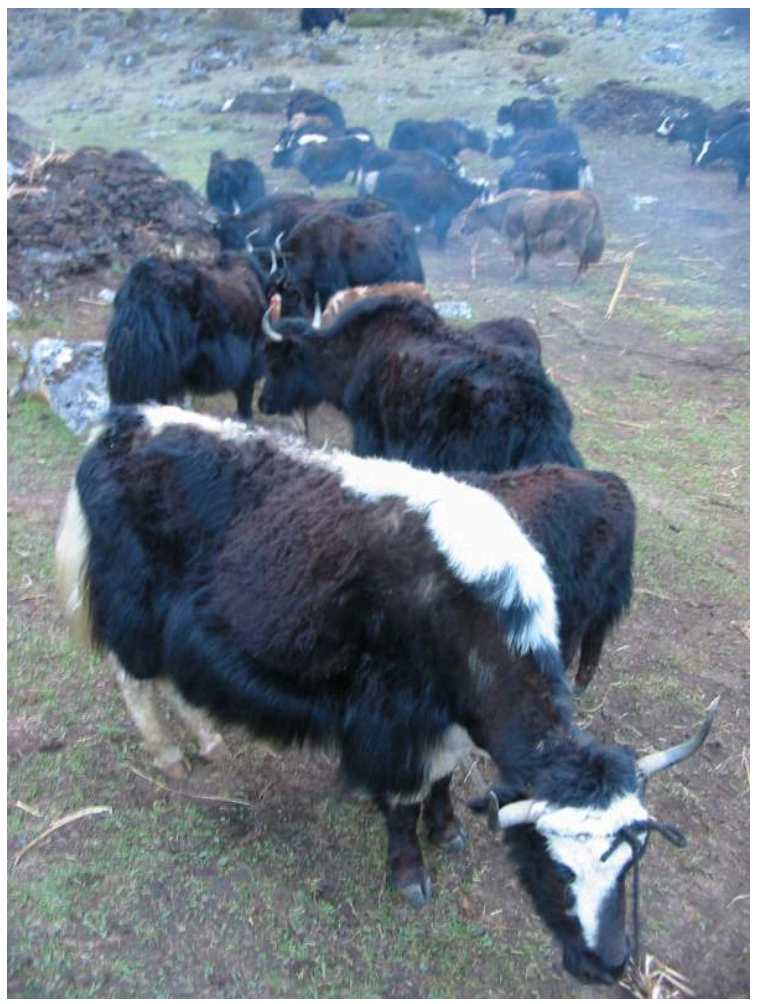

Dans ce système les veaux sont libres, les femelles sont attachées tous les soirs jusqu'à la traite du matin

Marie Dervillé (fin mai 2007, dans le geog de Linghzi, dans le Dzongkhag de Thimphu, altitude approximative $5200 \mathrm{~m}$ )

\section{Une transformation manuelle des produits}

La fabrication du beurre et du fromage à partir du lait $(90 \%$ du volume trait, les $10 \%$ restant étant consommés sous forme de thé au lait sucré) est manuelle. Elle a lieu au sein de chaque foyer. Le caractère extensif de l'élevage et l'absence de routes n'a pas, en effet, permis à ce jour de concentrer la production ou de réaliser des économies d'échelle, comme cela a pu être le cas en Chine.

Le lait n'est pas transformé immédiatement après la traite ; le lait est stocké jusqu'à ce qu'une quantité suffisante soit atteinte et permette de procéder à la transformation. Ce report permet une maturation du lait qui favorise sa transformation. L'extraction $\mathrm{du}$ beurre est réalisée dans la majorité des cas à l'aide d'une baratte à piston en bois (photo 7). 
Photo 7. Éleveuse de yaks du système Centre barattant le lait de yak dans une baratte à piston en bois

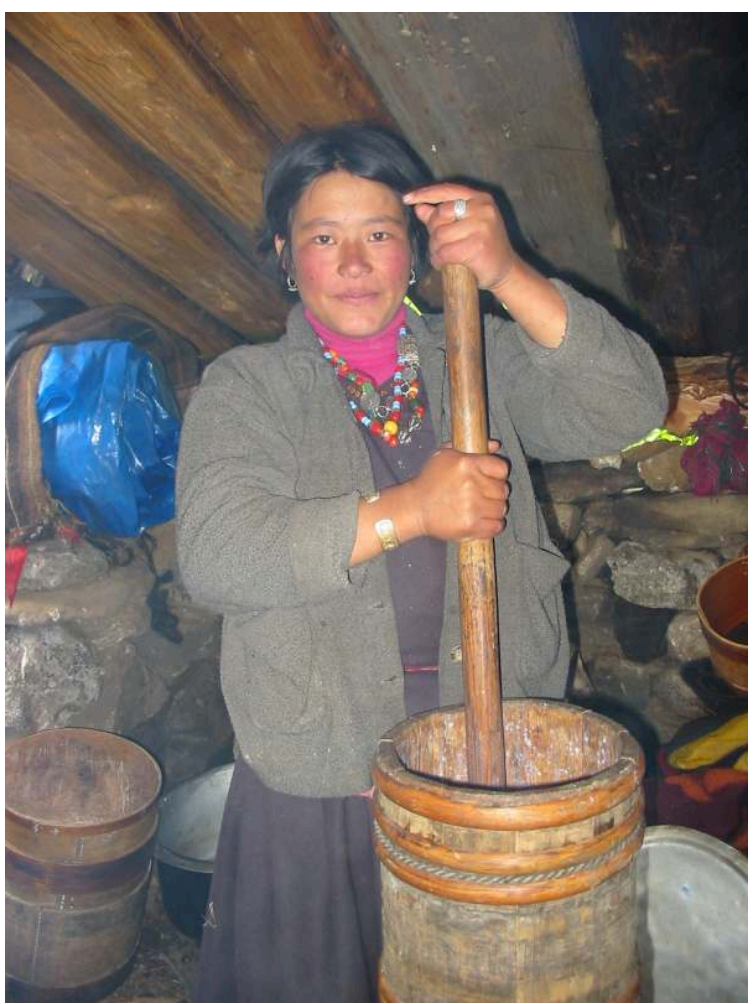

Marie Dervillé (juin 2007, sur le chemin de Gangar Punsum, vers les pâturages d'altitude, dans le geog de Chokhor, dans le Dzongkhag de Bhumthang, altitude approximative 4500 m)

Dans la région de Haa, toutefois, les éleveurs se servent d'une peau de jeune yak séchée comme récipient, qu'ils agitent à mouvements lents et réguliers au coin du feu. A l'est du pays enfin, des écrémeuses portatives sont de plus en plus utilisées (photo 8). 
Photo 8. Mère et fille utilisant une écrémeuse alpha laval dans le système Est

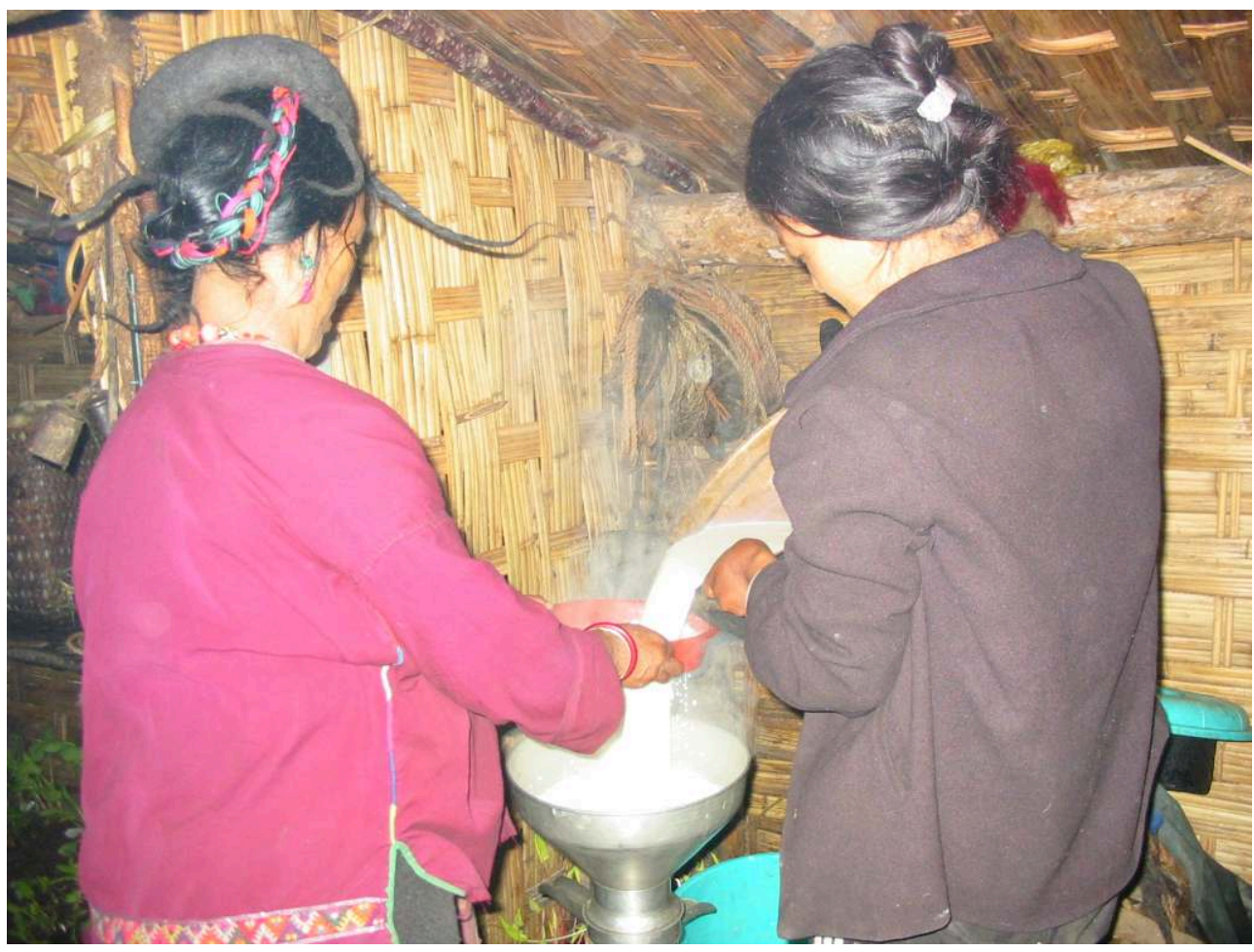

Marie Dervillé (juin 2007, dans le geog de Merak, dans le Dzongkhag de Trashigang, altitude approximative $3400 \mathrm{~m}$ )

Les éleveurs disposent souvent de barattes de deux tailles: un jeu adapté à la production d'hiver, l'autre à la production d'été. La durée de barattage varie avec la quantité, la température et la technique utilisée, entre une et trois heures. Après floculation, les particules de beurre sont rassemblées et pressées manuellement afin d'extraire le maximum d'eau, clé de la conservation du beurre. Le beurre est stocké de façon temporaire dans une boîte en bois. Le beurre destiné à la consommation et le beurre destiné à la commercialisation sont gérés séparément. Le beurre de commercialisation est constitué en blocs de deux à trois kilogrammes. Une fois qu'une quantité suffisante de beurre de surplus est mise de côté ( $40 \mathrm{~kg}$ en moyenne), les blocs de beurre sont rassemblés et empaquetés dans un sac en cuir. Le beurre peut ainsi se conserver plus d'un an. Il sera toutefois le plus fréquemment sorti et ré-emballé dans des feuilles, un tissu ou du papier journal, selon les localités, pour la commercialisation à la fin de l'automne. Les techniques de fabrication du beurre sont comparables à celles mises en évidence par Dong sur le plateau du Quinghai-Tibet (Dong et al. 2003).

Après extraction du beurre, le babeurre est chauffé à feu doux. Du babeurre fermenté est souvent ajouté pour accélérer le processus de coagulation. Après caillage, le fromage est extrait et égoutté quelques heures, le plus souvent par pressage entre deux pierres. Selon les régions, le fromage issu du babeurre est soit consommé ou commercialisé en frais ou demi sec, soit, pour une plus grande part, transformé en fromage fermenté (à l'est du pays) ou en fromage séché (Haa, Ouest, Centre).

La fabrication de fromage sec et demi-sec à partir du babeurre est également pratiquée sur le plateau tibétain mais la production de fromage fermenté semble une spécificité 
du système d'élevage de l'est du Bhoutan. Ce fromage après égouttage est fourré dans un sac de peau. La maturation peut durer un an.

Le fromage destiné à la production de fromage séché est découpé, après égouttage, en morceaux de taille variable selon le type de fromage séché. Ces morceaux sont ensuite enfilés sur un fil en poils de yak ou plus récemment en nylon (photo 9) :

- dans le cas du fromage séché blanc de petite taille (churghu), les morceaux ont pour dimension $1 \times 1 \times 4 \mathrm{~cm}$ ou $1 \times 3 \times 3 \mathrm{~cm}$

- dans le cas du fromage séché noir de grande taille (hapi ruto), les morceaux mesurent $1 \times 8 \times 8 \mathrm{~cm}$.

Photo 9. Éleveuse de yaks du système Centre enfilant des dés de fromage sur un fil de nylon

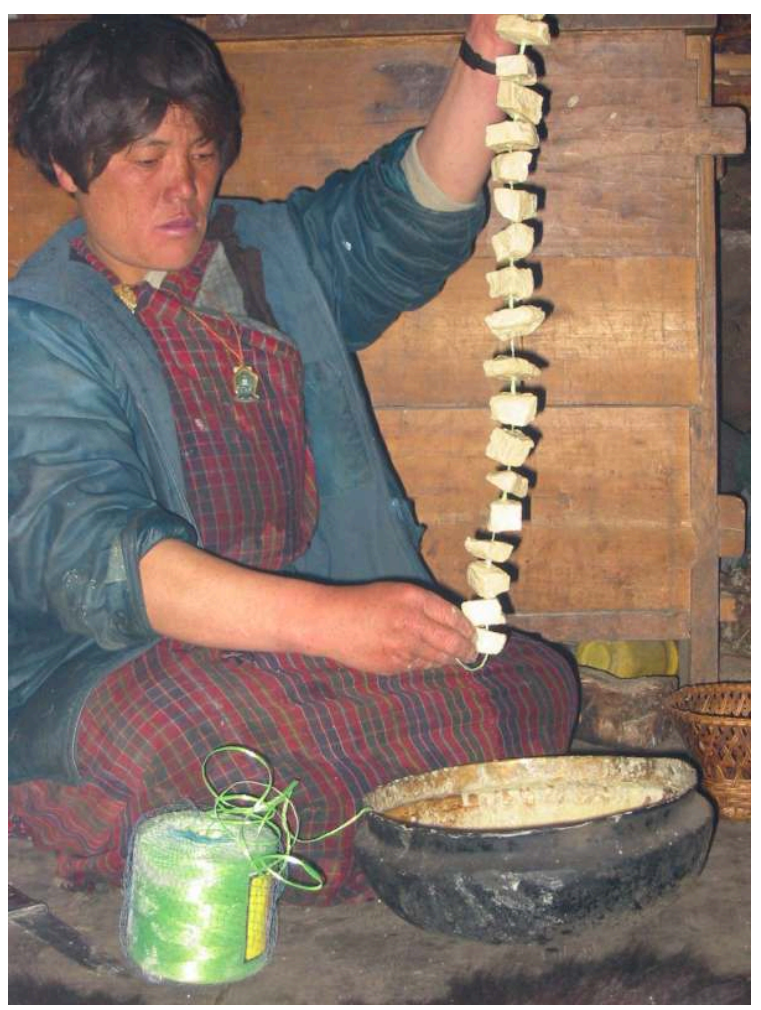

Ces colliers de fromages sècheront à l'air libre avant d'être mis en sac et commercialisés à l'automne Marie Dervillé (juin 2007, sur le chemin de Gangar Pensuem dans le geog de Chokhor, dans le Dzongkhag de Bhumthang, altitude approximative $4500 \mathrm{~m}$ )

Le processus de fabrication de ces deux types de fromage séché diffère: pour la production de fromage séché blanc, les petits cubes de fromage sont bouillis une deuxième fois dans du lactosérum ou dans du lait - ce qui leur confère un goût sucré prisé des consommateurs - avant d'être séchés de préférence au soleil ou, lorsque le temps ne le permet pas, à l'intérieur, au coin d'un feu de bois. La combustion de bouses de yaks noircit en effet les fromages. Le noircissement est recherché pour les fromages séchés noirs de grande taille mais réduit fortement la valeur marchande du fromage séché blanc de petite taille. Dans la région de Haa, la rareté du bois sur les pâturages d'altitude explique que la fabrication de fromage séché se limite aux fromages séchés noirs de grande taille. 


\section{Une activité familiale}

L'élevage est majoritairement pratiqué en famille. Deux générations collaborent la plupart du temps : deux couples - les parents et les enfants - ou des parents et de jeunes adultes. La taille moyenne de la famille impliquée dans la gestion du troupeau est de sept personnes : trois ou quatre personnes actives, une à deux personnes âgées plus ou moins inactives, deux enfants de moins de 15 ans. La répartition des tâches entre homme et femme est variable, à l'exception de l'activité de commerce réservée aux hommes. Les femmes s'occupent fréquemment de la traite et des fabrications laitières, mais de façon non exclusive.

En moyenne sur l'année, un peu plus de $60 \%$ du temps est consacré à la conduite du troupeau (surveillance, accompagnement des vêlages, suivi sanitaire, alimentation), près de $20 \%$ à la traite et à la fabrication, $5 \%$ au commerce. Un peu plus de $10 \% \mathrm{du}$ temps est consacré à des activités ponctuelles : migration (cinq à douze jours selon les systèmes), peignage de la bourre et coupe des poils au printemps, récolte de fourrages pour l'hiver, ramassage de bois (figure 3).

Figure 3. Répartition annuelle du travail par bloc d'activité

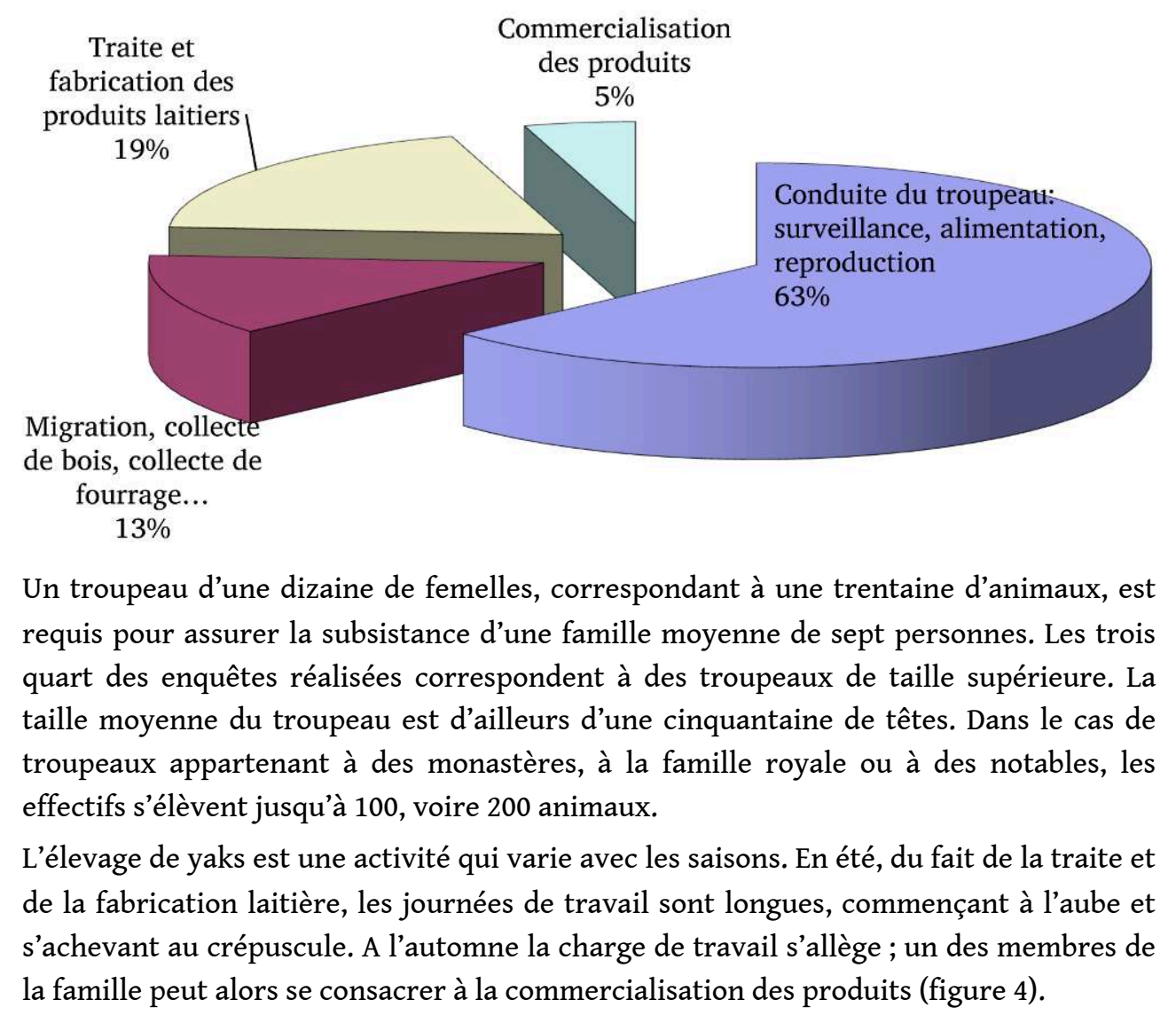

Études mongoles et sibériennes, centrasiatiques et tibétaines, 43-44 | 2013 
Figure 4. Calendrier de travail

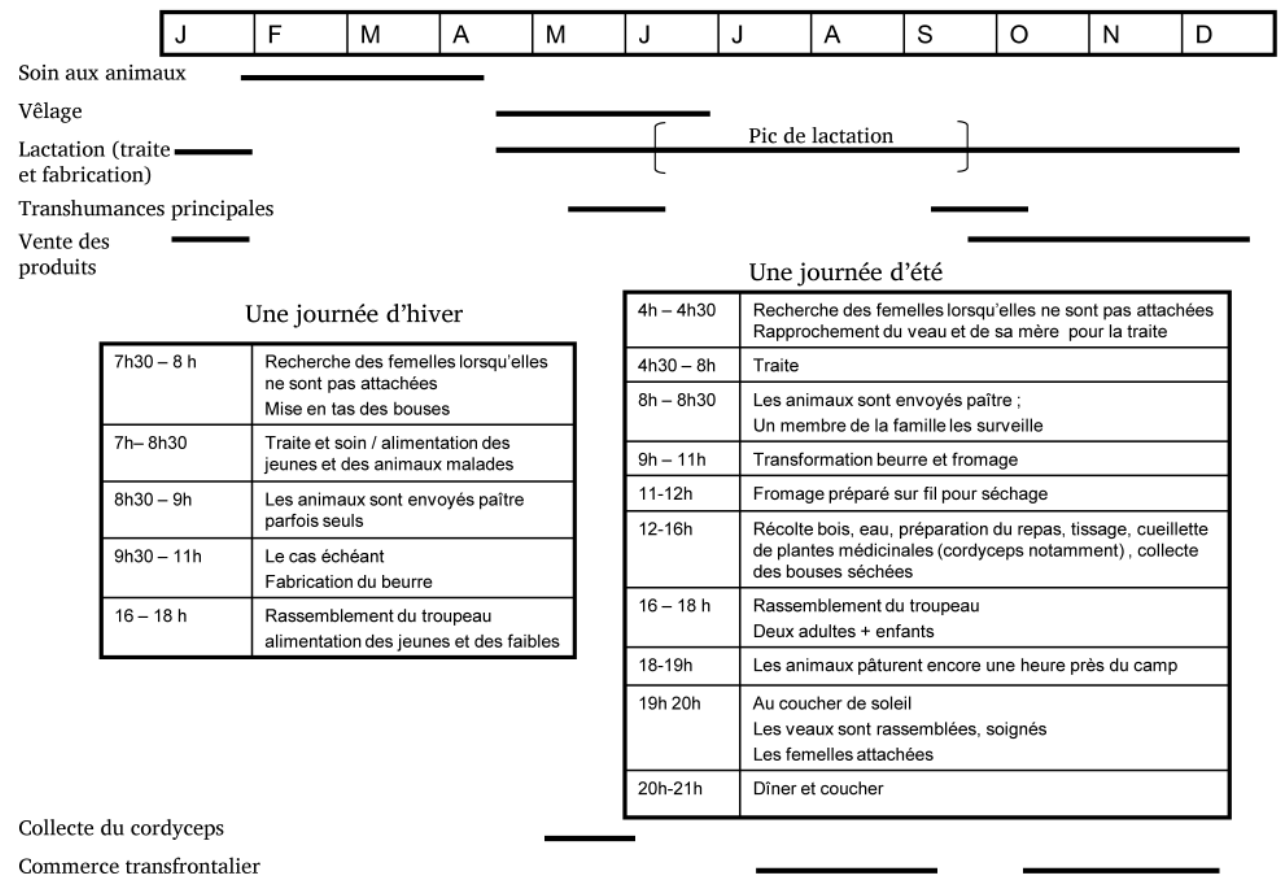

Dans certaines régions comme celles de Bumthang et de Haa, les éleveurs bénéficient, au travers de la famille élargie ${ }^{5}$ qui possède des terres agricoles en zone périurbaine, d'un accès privilégié aux services (accès à l'éducation pour les enfants notamment) et aux opportunités économiques nouvelles (commerce de proximité, emploi de chauffeur, etc.). Il en résulte une raréfaction de la main d'œuvre disponible pour l'élevage. Une gestion a minima de l'organisation du travail s'est de ce fait développée. Deux à trois personnes à temps plein en été et une personne en hiver sont mobilisées pour la conduite du troupeau et la transformation des produits.

\section{Une valorisation des produits reposant traditionnellement sur deux types de réseaux d'échange}

L'élevage de yaks est essentiellement une activité de subsistance.

L'auto-consommation moyenne de laitages, en équivalent lait, est comprise entre 95 et $155 \mathrm{~kg}$ par personne ${ }^{6}$ selon les régions. Elle est la plus faible dans les régions de Thimphu et Paro ainsi qu'à l'est du pays (région de Trashigang). Elle est maximale dans la région de Haa. La consommation de produits laitiers se retrouve ainsi à un niveau inférieur à celui des pays européens - $465 \mathrm{~kg} / \mathrm{p} / \mathrm{an}$ au Danemark, $400 \mathrm{~kg} / \mathrm{p} / \mathrm{an}$ en France et environ $300 \mathrm{~kg} / \mathrm{p} / \mathrm{an}$ au Royaume Uni et en Italie (CNIEL 2009) - mais proche du niveau de consommation en Mongolie estimé par la FAO à $140 \mathrm{~kg} / \mathrm{p} / \mathrm{an}$ (FAO 2006) et supérieur au niveau de consommation de laitage dans les villes chinoises (56 et $57 \mathrm{~kg}$ / $\mathrm{p} / \mathrm{an}$ à Shanghaï et Pekin) (Mission Economique de Pékin 2006). Si les éleveurs de yaks consomment du lait dans leur thé sucré (naja), la principale forme de consommation du lait est sous forme de beurre utilisé dans la cuisine mais aussi et surtout dans la préparation du thé salé (suja). Le fromage est consommé frais en sauce en accompagnement d'un plat de riz. A l'est, les éleveurs consomment le fromage fermenté sous forme de soupe. 
41 La viande destinée à la consommation des éleveurs provient de deux sources : mort accidentelle des animaux ou abattage. Pour être conservée, la viande est séchée au soleil ou fumée. Une sorte de boudin (jooma) est également réalisée à partir du sang. Une partie des produits est commercialisée pour permettre, notamment, l'approvisionnement en céréales de la famille. La part de production vendue varie avec la taille et le statut (en propriété ou pas) du troupeau. Elle varie aussi avec les produits : moins de $45 \%$ pour la viande et le beurre et plus de $70 \%$ pour le fromage, en moyenne (fig. 5). Le fromage séché, destiné principalement à la commercialisation ou à la réalisation de présents, est en effet peu autoconsommé.

Figure 5. Entre autoconsommation et commercialisation : devenir des différents produits issus de l'élevage de yaks

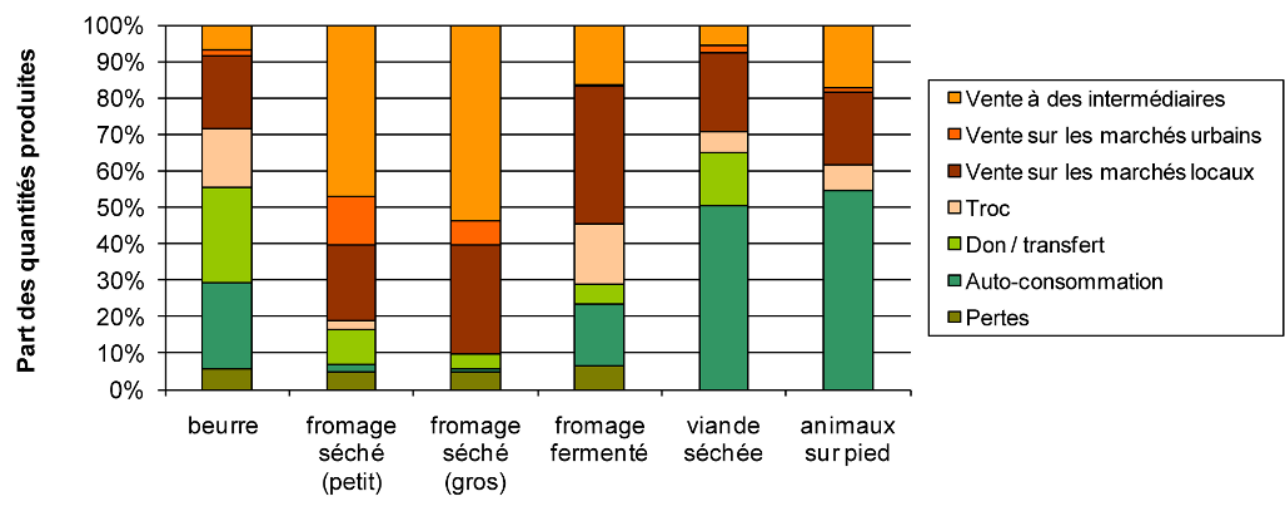

43 La modalité historique d'échange est le troc : le beurre, le fromage, la viande séchée et les animaux étaient traditionnellement échangés contre des céréales, du sel, des ustensiles et des vêtements. Bien que sur le déclin, cette pratique persiste encore aujourd'hui surtout pour le beurre et la viande séchée. La commercialisation des animaux pour l'abattage et celle des fromages séchés se sont en revanche progressivement monétisées.

Les échanges de viande séchée, de beurre et, dans une moindre mesure, de fromages se déroulent dans le cadre de deux types de relations sociales non exclusives: des liens d'interdépendance avec les grands propriétaires de yaks (seigneurs ou monastères) et des liens d'entraide avec les agriculteurs de basse altitude.

Jusqu'à l'abolition du système de taxation en nature (1956), l'élevage était fortement taxé. Nombre d'éleveurs préféraient alors vendre leurs services de vacher auprès des grands propriétaires nobles ou religieux que de posséder un troupeau. Dans les années 1960, la moitié du cheptel appartenait ainsi à des monastères ou à de grands propriétaires (Dr Pema Gyamthso ${ }^{7}$, communication personnelle, 2009). Si la fréquence de ces arrangements est aujourd'hui moindre, elle reste structurante à l'ouest et au centre du pays. L'éleveur et sa famille sont en charge du troupeau et de la fabrication laitière. Ils reçoivent en échange une partie des produits (animaux, beurre et surtout fromage) ainsi qu'une ration annuelle de céréales et des vêtements. Le propriétaire pouvait également se charger de commercialiser des produits de l'éleveur avec les siens. Deux principaux types d'arrangement existent entre propriétaire et éleveur :

- un transfert fixe de produits skyesmed chimed $^{8}$ : ce type d'arrangement signifie que le nombre de yaks du propriétaire reste inchangé quelles que soient les naissances ou les pertes.

L'éleveur peut ainsi se constituer un troupeau à partir des naissances les années fastes mais, 
en cas de taux de mortalité élevé, il devra remplacer les animaux du propriétaire par les siens. Dans ce type d'arrangement, la quantité de beurre transférée au propriétaire est indépendante des vêlages, elle est fixée en général à 1,3 kg de beurre par animal (tous animaux du troupeau confondus).

- un transfert de produits proportionnel au nombre de vêlages skyesyod 'chiyod, qui correspond à une relation de métayage au sens commun du terme, avec partage des fruits et des pertes. Les veaux sont partagés entre le métayer et le propriétaire et les prélèvements, concernant la production de beurre uniquement, sont proportionnels aux vêlages : 4 à $5 \mathrm{~kg}$ de beurre par femelle en lactation et par an sont transmis au propriétaire, ce qui représente environ $60 \%$ de la production moyenne annuelle de beurre. une transaction ponctuelle. Il s'agit d'un partenariat de longue durée avec une « famille hôte de basse altitude ». Cette dernière, non seulement offre le gîte et le couvert à l'éleveur lorsqu'il descend dans la vallée, mais elle l'aide à commercialiser ses produits. Éleveur de yaks et hôte font du porte à porte dans la vallée pour placer le beurre, le fromage et la viande séchée. Une fois la promesse d'échange scellée, l'éleveur rejoint son troupeau. C'est l'hôte qui rassemblera la quantité de céréales due à l'éleveur après la récolte. Dans certaines régions comme celles de Bumthang ou de Haa, c'est la famille élargie propriétaire de terres agricoles de basse altitude qui se charge de la commercialisation des produits. Avant la sédentarisation de l'élevage bovin laitier dans les vallées, le beurre de yak, outre son usage religieux et festif, était considéré par les consommateurs comme un beurre d'hiver : un substitut au beurre de vache lorsque les troupeaux bovins avaient migré des zones tempérées aux zones tropicales. Ces dernières années, l'échange de fromages est devenu de plus en plus marchand. Des grossistes viennent à la rencontre des éleveurs à l'automne et achètent la totalité de leur production fromagère, pour la revendre en Inde ou sur les marchés bhoutanais urbains. Certains éleveurs démarchent également individuellement les commerces des villes et des villages à proximité de leur campement d'hiver. Certains cherchent aussi à commercialiser directement leur produit sur le bord des routes.

La commercialisation des animaux est également largement devenue marchande. Les animaux destinés à l'abattage sont descendus à l'automne lorsque leur état est le meilleur. Les éleveurs vendent les animaux sur pied à des intermédiaires, des bouchers, ou des particuliers. Des relations de confiance inscrites dans la durée peuvent exister mais les liens sociaux sont moins forts que dans le cas de l'échange de beurre. L'abattage est réalisé (à l'aide d'une dague enfoncée en plein cœur) par des Bhoutanais d'origine népalaise ou tibétaine, moins sujets à la pression bouddhique ou contraints par le besoin. Si les bouchers disposent d'une boutique, la majorité des intermédiaires vendent la viande au détail sur la place du marché (photo 10). 
Photo 10. Vente de viande de yak sur la place du village de Haa

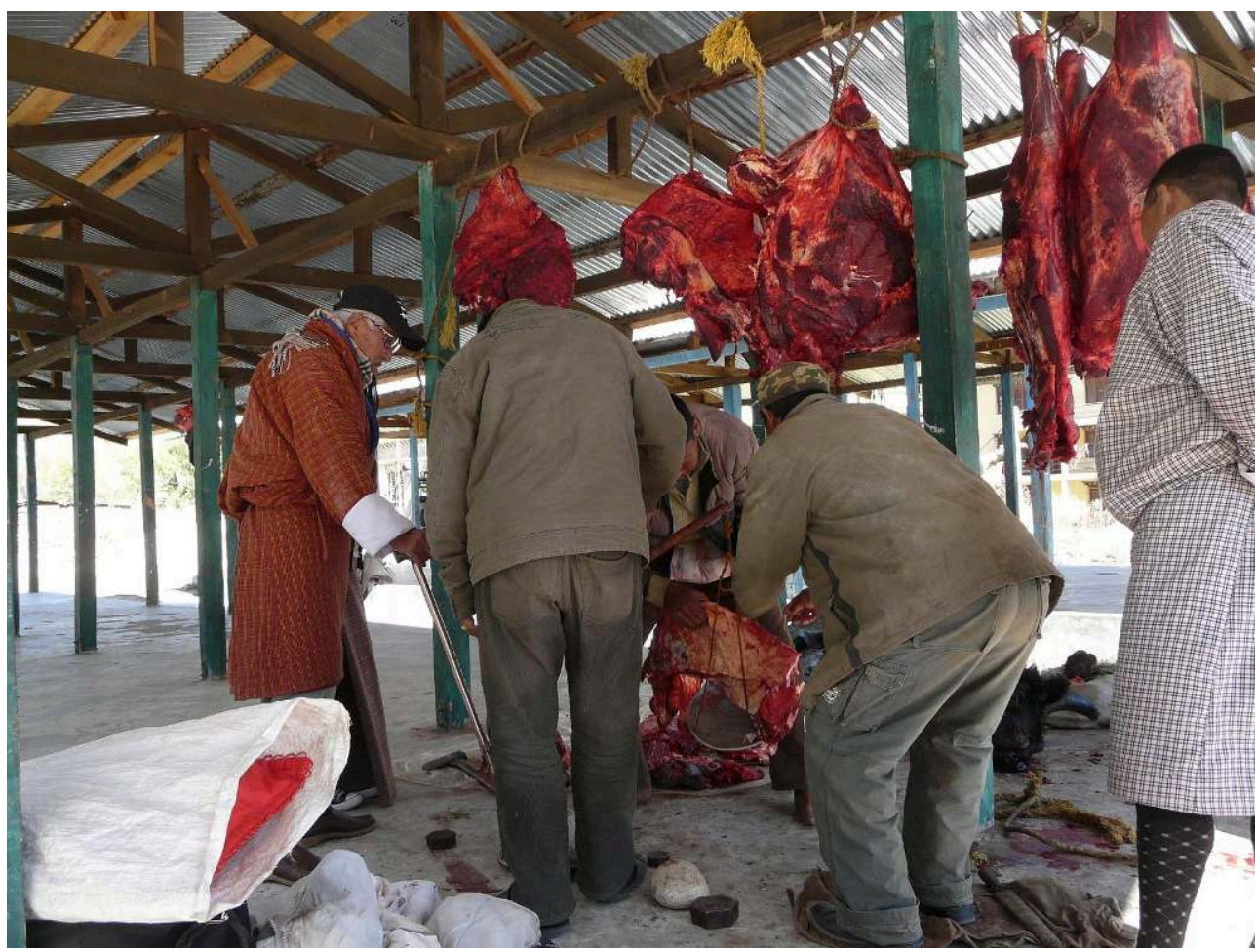

Des intermédiaires ont acheté un yak sur pied à un éleveur, avant de le faire abattre et de le commercialiser. La commercialisation d'un yak entier peut prendre 2-3 jours

Marie Dervillé (octobre 2009, dans le geog de Sama, dans le Dzongkhag de Haa, altitude approximative $3000 \mathrm{~m}$ )

La caractérisation des pratiques de conduite et d'exploitation a permis l'estimation des performances technico-économiques de l'activité d'élevage de yaks au Bhoutan. Ces performances sont territorialement contrastées en relation avec la diversité des pratiques et les modalités d'insertion sociale des éleveurs qui conditionnent le niveau de valorisation des produits. Je propose de définir quatre systèmes régionaux d'élevage. La viabilité de l'activité d'élevage peut ainsi être discutée au regard des écarts inter- et intra-système de revenus. Enfin, je présente ici une première lecture des dynamiques d'élevage spatialement contrastées est présentée.

\section{Des performances technico-économiques fortement contrastées selon les régions et à l'intérieur de celles- ci}

\section{Une productivité limitée}

Comme on le verra ci-dessous, les performances technico-économiques rapportées par les éleveurs sont en accord avec la littérature (Wiener et al. 2003), quoique dans la fourchette basse.

Les femelles vêlent pour la première fois à quatre ans et ensuite tous les deux ans. Ces résultats, conformes à ceux qui sont consignés dans les travaux spécialisés, pourraient être améliorés avec une meilleure alimentation : le premier vêlage pourrait avoir lieu à 
trois ans au lieu de quatre et l'intervalle entre deux vêlages pourrait être réduit à un an (G. Wiener, communication personnelle, 2010).

51 Au pic de lactation, en juillet-août, la traite des femelles yaks de race pure donne en moyenne 0,75 à 1,5 $\mathrm{l}$ par jour. De mi-septembre à décembre, la traite se limite à $20-30 \mathrm{cl}$ par jour. Ensuite la totalité de la production est laissée au veau. La traite reprendra au printemps suivant jusqu'au milieu de l'été, date de tarissement des femelles pleines. Ces chiffres conformes aux statistiques publiées puisque la production de lait (incluant la consommation du veau qui est estimé à la moitié de la production dans le cas d'une traite par jour) varie selon les auteurs entre 0,8 1 (Ju Quimei et al. 2000, tiré de Wiener et al. 2003) et 31 par jour (Zhang Rongchang 1989, tiré de Wiener et al. 2003). Ainsi la quantité traite par lactation (sur deux ans) semble varier entre 160 et 300 l. de lait, avec une valeur moyenne proche de 2001.

Le taux de transformation du lait est compris entre 13 et $16 \mathrm{l} / \mathrm{kg}$ de beurre. Le taux de matière grasse du lait de yak ( $7 \%, c f$. Karma Ura 1993) explique ce rendement élevé. La fabrication d'1 $\mathrm{kg}$ de beurre s'accompagne de la fabrication d'environ 2 à $3 \mathrm{~kg}$ de fromage frais, soit $1,5 \mathrm{~kg}$ de fromage séché, ou de $2 \mathrm{~kg}$ de fromage fermenté.

La traite des femelles hybrides dzomo peut atteindre 31 par jour au pic de lactation, soit trois fois plus que les femelles de race pure. Mais cette pratique de l'hybridation modifie la conduite du troupeau puisque la plupart des éleveurs ne gardent que les hybrides de première génération; les hybrides mâles $d z o$ sont d'ailleurs stériles. Par ailleurs, les hybrides ont une adaptation à l'altitude intermédiaire entre celle des bovins et des yaks; ils valorisent mieux un étage écologique particulier.

Les taux de mortalité rapportés par les éleveurs sont élevés : entre 18 et $31 \%$ de perte sur les jeunes animaux de moins de deux ans, entre 23 et $44 \%$ sur les animaux de deux à quatre ans et 8 à $13 \%$ sur la vie d'adulte. Les causes principales sont les accidents, les maladies (Cœnurose ${ }^{9}$ notamment), la malnutrition, les attaques par des animaux sauvages. Dans ces conditions, un troupeau de 10 femelles ( 5 vêlages par an) permet à peine de vendre un animal par an. Le poids des animaux abattus au Bhoutan est estimé entre 250 et $300 \mathrm{~kg}$ par les bouchers et le service sanitaire. En prenant un rendement en viande moyen de 50-55\%, j'ai considéré des carcasses de $150 \mathrm{~kg}$. Les sources écrites rapportent des chiffres comparables avec un poids variant de 116 à $576 \mathrm{~kg}$ en fonction de la race et de la localisation avec un rendement en carcasse compris entre 40 et $62 \%$ (Wiener et al. 2003).

Les animaux jeunes, de trois à six ans, essentiellement des mâles, sont également commercialisés occasionnellement en tant que reproducteurs. Cette pratique est particulièrement fréquente dans la région de Haa, reconnue pour la qualité génétique de son cheptel.

La production de poils, prélevés chez tous les animaux adultes, est estimée à $0,5 \mathrm{~kg}$ par animal et la production de bourre, prélevée sur les animaux de moins de trois ans, à moins de $0,5 \mathrm{~kg}$ par animal. Cette pratique, faute de débouché, est toutefois sur le déclin.

Résultant des pratiques de conduite et d'exploitation, le troupeau se compose en moyenne d'environ 30 à $40 \%$ de femelles dont une petite moitié vêle chaque année, de 15 à $30 \%$ de mâles selon les régions, en lien avec les pratiques d'abattage, et d'un gros tiers de jeunes animaux de moins de quatre ans. 


\section{Une diversité régionale des systèmes d'élevage de yaks bhoutanais}

Mon objectif est de mettre en évidence, au-delà de la diversité individuelle, des invariants qui permettent de caractériser les systèmes d'élevage et de les différencier sur une base territoriale. La conduite de la reproduction (avec ou sans hybridation), les pratiques d'exploitation (avec ou sans abattage), ainsi que les pratiques de valorisation (type de produits et marchés), semblent les plus discriminantes dans l'élevage de yaks bhoutanais. C'est autour d'elles, du statut du troupeau et des pâturages (en métayage, en propriété, ou communautaires) et de la localisation géographique que quatre grands systèmes d'élevage ont pu être distingués (Dervillé \& Bonnemaire 2010). Avec d'ouest en est (fig. 6 et 7) :

- le SYSTEME de HAA concerne trois geogs de la région de Haa, $7 \%$ de la population de yaks et 141 foyers ${ }^{10}$ d'éleveurs. Ce système est caractérisé par un éloignement important (jusqu'à huit jours de marche) entre campements d'hiver et campements d'été. Les campements d'hiver sont situés à l'extrémité de la vallée de Haa, à proximité de la route et des terres agricoles de la famille élargie, à environ $3000 \mathrm{~m}$ d'altitude. Les campements d'été sont situés en bordure de plateau tibétain à la frontière avec la Chine, à plus de 5000m. L'élevage est pratiqué en race pure. Les pâturages sont communautaires. Les principaux produits sont les animaux pour l'abattage ou la reproduction, le beurre et le fromage séché. L'élevage de yaks est complémentaire de l'élevage bovin laitier et de la culture du blé et du radis. Le marché traditionnel est Paro pour le beurre et la viande et l'Inde pour le fromage séché.

- le SYSTEME OUEST recouvre 13 geogs des régions de Paro, Thimphu et Gasa. On y trouve $51 \%$ de la population de yaks et 345 foyers. L'élevage de yaks, pratiqué en race pure, est l'activité principale des éleveurs. Les habitations principales des éleveurs sont construites à plus de $4000 \mathrm{~m}$ d'altitude. L'habitat est groupé mais éloigné des axes de communication; les villages ainsi constitués se situent à trois jours ou plus de marche de la route la plus proche. Les campements d'été sont relativement accessibles en rayonnant autour de ces villages, de quelques heures à deux jours de marche. Les vallées sont larges, les paysages sont ouverts. La collecte de plantes médicinales et le commerce transfrontalier ${ }^{11}$ constituent également des sources de revenu. Une part importante des cheptels et des droits de pâturage ( $45 \%$ des éleveurs rencontrés étaient concernés en 2007) appartient à des monastères. Thimphu et Paro sont les principaux débouchés pour le beurre, la viande et le fromage.

- le SYSTEME CENTRE correspond à neuf geogs des régions de Wangdue et Bumthang où se trouvent $16 \%$ de la population de yaks et 120 foyers. Dans cette région, l'élevage de yaks est une activité secondaire, complémentaire de la culture de pomme de terre, de l'élevage bovin et du travail du bois. À l'instar du système de Haa, l'éloignement est marqué entre le campement d'hiver de plus basse altitude $(\sim 3000 \mathrm{~m})$ et les pâturages les plus élevés $(\sim 500 \mathrm{~m})$. Cependant, la migration est progressive, de nombreuses pauses de plusieurs semaines sont effectuées à l'aller et au retour. L'hybridation avec les bovins était historiquement pratiquée : la force de traction des hybrides mâles était recherchée. Cette pratique a quasiment disparu avec le développement de la motorisation. Du fait de sentiments religieux très prégnants, les yaks ne sont pas (Bumthang) ou peu (Wangdue) abattus dans cette zone.

- le SYSTEME EST s'étend sur six geogs des régions de Luentshe, Tashi Yangtse, Trashigang et Samdrup Jonkhar. Un quart de la population de yaks (dont ici la moitié est constituée d'hybrides) appartient à ce système. 395 foyers sont concernés. Yaks et hybrides sont conduits séparément, les pâturages d'été des hybrides correspondant souvent aux pâturages d'hiver des yaks. L'habitat est groupé ; la région de Trashigang compte deux principales 
zones de peuplement réunissant la majorité des foyers : les villages de Merak et de Sakteng situés approximativement à 3400 et $2950 \mathrm{~m}$ d'altitude respectivement. Ces villages sont relativement proches des axes de communication (une journée de marche avec les animaux, une demie sans eux). De ce fait, les échanges avec les agriculteurs de basse altitude, voire avec les habitants de la principale ville de la région sont fréquents : les échanges de produits laitiers contre des céréales sont réalisés en plusieurs fois, les éleveurs effectuant en moyenne une dizaine de voyages par an. Cette spécificité permet la commercialisation d'une partie du fromage de femelles hybrides en frais. Les hybrides mâles sont quant à eux mis à profit pour leur force de traction (photo 11). Une autre spécificité de ce système réside dans la persistance de l'élevage ovin, encore pratiqué par près de la moitié des éleveurs, alors qu'il a quasiment disparu dans le reste du pays. Les échanges commerciaux avec l'État de l'Arunachal Pradesh, en Inde sont fréquents.

Figure 6. Carte écologique du Bhoutan et typologie des systèmes d'élevage de yaks

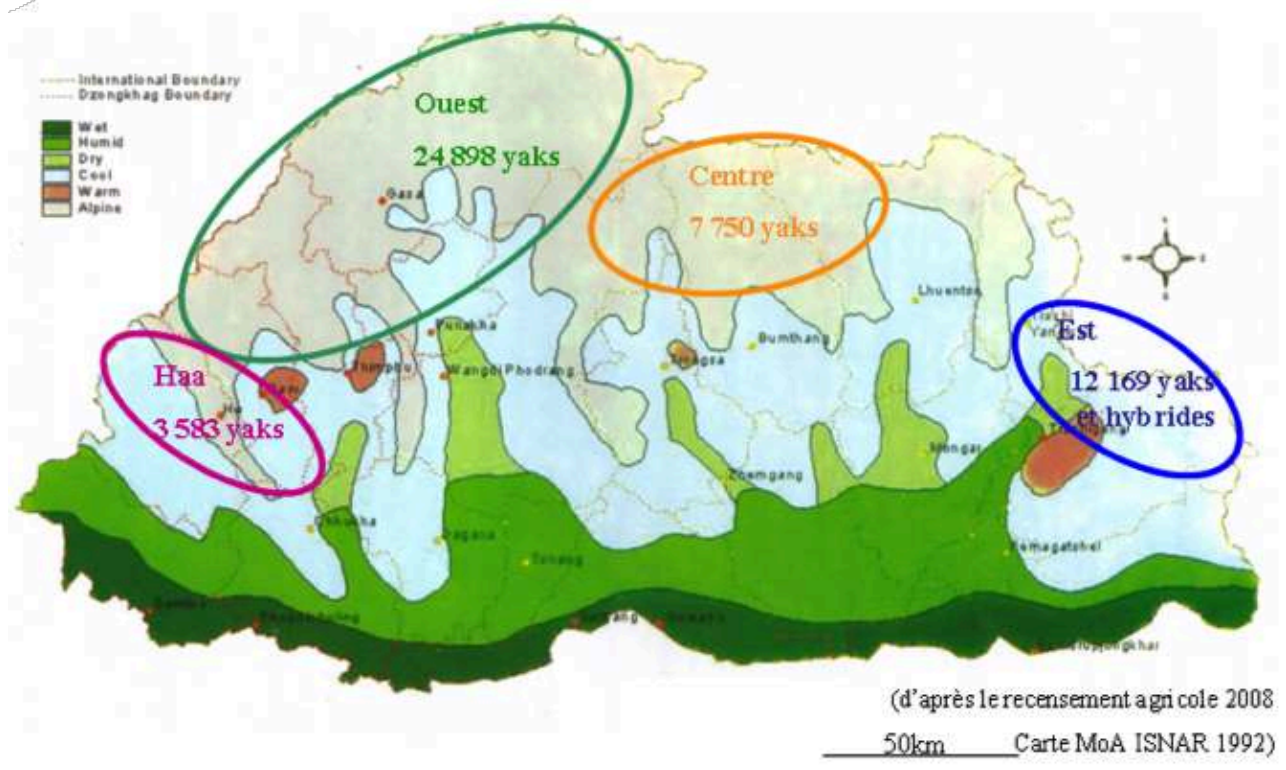


Figure 7. L'élevage des yaks au Bhoutan : caractéristiques des 4 systèmes régionaux d'élevage

\begin{tabular}{|c|c|c|c|c|c|}
\hline Système & Troupeau & Accès au pâturage & Equipement & Produits & Diversification \\
\hline $\begin{array}{l}\text { Haa } \\
-3 \text { geogs } \\
-141 \text { foyers } \\
-3583 \text { yaks } \\
-0 \text { hybride } \\
13 \text { enquêtes }\end{array}$ & \begin{tabular}{|l|} 
- Yaks en \\
propriété \\
$(86 \%)$ \\
-52 yaks en \\
moyenne
\end{tabular} & $\begin{array}{l}\text { - Pâturage } \\
\text { communautaire joué } \\
\text { aux dés } \\
\text { - Pâture en bon état } \\
\text { - Conflit avec } \\
\text { tibétains pour } \\
\text { pâtturage d'été }\end{array}$ & $\begin{array}{l}\text { - Baratte en peau } \\
\text { - Maison à la } \\
\text { farmille élargie, } \\
\text { - habitat } \\
\text { sommaire (bois ou } \\
\text { pierre) }\end{array}$ & $\begin{array}{l}\text { - Animaux sur pied et } \\
\text { reproducteurs } \\
\text { - Viande séchée } \\
\text { - Beuture et fromage } \\
\text { séché (gros) } \\
\text { - Fromage fermenté (lait } \\
\text { entier) } \\
\text { Marché principal : Paro }\end{array}$ & $\begin{array}{l}\text { - Surfaces } \\
\text { agricoles de basse } \\
\text { altitude } \\
\text { - Commerce avec } \\
\text { le Tibet }\end{array}$ \\
\hline $\begin{array}{l}\text { Ouest } \\
-13 \text { geogs } \\
-345 \text { foyers } \\
-24898 \text { yaks } \\
-0 \text { hybride } \\
29 \text { enquêtes }\end{array}$ & \begin{tabular}{|l|} 
- Yaks en \\
propriété \\
$(78 \%)$ \\
-59 yaks en \\
moyenne
\end{tabular} & $\begin{array}{l}\text { - } 45 \% \text { des droits de } \\
\text { pâturage } \\
\text { appartiennent à des } \\
\text { monastères } \\
\text { - Pâturage dégradé } \\
\text { en de nombreux } \\
\text { endroits }\end{array}$ & $\begin{array}{l}\text { - Baratte à piston, } \\
\text { - Panneau solaire } \\
\text { - Maison en dur } \\
\text { sur le campement } \\
\text { d'hiver }\end{array}$ & $\begin{array}{l}\text { - Animaux sur pied } \\
\text { - Beurre et fromage } \\
\text { séché (petit et gros) } \\
\text { - Fromage fermenté (lait } \\
\text { entier) } \\
\text { - Sacs, lentes, cordes } \\
\text { Marché principal : Paro, } \\
\text { Thimphu }\end{array}$ & $\begin{array}{l}\text { - Commerce avec } \\
\text { le Tibet } \\
\text { - Cordyceps } \\
\text { - Plantes } \\
\text { médicinales } \\
\text { - Tourisme } \\
\text { - Achat de terre }\end{array}$ \\
\hline $\begin{array}{l}\text { Central } \\
-9 \text { geogs } \\
-120 \text { foyers } \\
-7750 \text { yaks } \\
- \text { dont }-50 \\
\text { hybrides } \\
17 \text { enquêtes }\end{array}$ & \begin{tabular}{|l|} 
- Yak en \\
propriété \\
$(85 \%)$ \\
-59 yaks en \\
moyenne
\end{tabular} & $\begin{array}{l}\text { - Pâturage en } \\
\text { communauté / } \\
\text { groupe d'usagers } \\
\text { ( } 35 \% \text { des éleveurs } \\
\text { enquêlés) } \\
\text { - Pâturage dégradé } \\
\text { autour des } \\
\text { campements }\end{array}$ & $\begin{array}{l}\text { - Baratte à piston } \\
\text { - Maison à la } \\
\text { famille élargie } \\
\text { - Baraquements } \\
\text { en bois ou en } \\
\text { pierre en altitude }\end{array}$ & $\begin{array}{l}\text { - Viande séchée } \\
\text { uniquement } \\
\text { - Beurre } \\
\text { - Fromage séché (petit) } \\
\text { - Fromage fermenté } \\
\text { - Sacs, tapis, cordes } \\
\text { Marché principal : } \\
\text { Bumthang }\end{array}$ & $\begin{array}{l}\text { - Surfaces } \\
\text { agricoles de basse } \\
\text { altitude, élevage } \\
\text { de mouton } \\
\text { - Cordyceps } \\
\text { - Tourisme } \\
\text { - Cormmerce avec } \\
\text { le Tibet } \\
\end{array}$ \\
\hline $\begin{array}{l}\text { Est } \\
-6 \text { geogs } \\
-395 \text { foyers } \\
-12169 \text { yaks } \\
- \text { dont } \sim 6000 \\
\text { hybrides } \\
26 \text { enquêtes }\end{array}$ & $\begin{array}{l}\text { - Yaks en } \\
\text { propriété } \\
\text { (93\%) } \\
-32 \text { yaks et } \\
\text { hybrides en } \\
\text { moyenne }\end{array}$ & $\begin{array}{l}\text { - Pâturage } \\
\text { communautaire de } \\
\text { qualité variable; } \\
\text { - Conflit avec les } \\
\text { éleveurs de la } \\
\text { vallée } \\
\text { - Tension avec le } \\
\text { parc naturel }\end{array}$ & $\begin{array}{l}\text { - Ecrémeuse } \\
\text { - Panneau solaire } \\
\text { - Maison en dur } \\
\text { sur le campernent } \\
\text { d'hiver }\end{array}$ & $\begin{array}{l}\text { - Animaux vers l'Inde } \\
\text { - Viande séchéé } \\
\text { - Beurre er fromage frais } \\
\text { - Fromage fermenté } \\
\text { - Sacs, tapis, cordes } \\
\text { Marché principal: } \\
\text { villages des vallées, } \\
\text { Trashigang }\end{array}$ & \begin{tabular}{|l} 
- Commerce avec \\
l'Inde \\
- Tissage \\
- Elevage de \\
mouton
\end{tabular} \\
\hline
\end{tabular}

Le nombre de foyers est issu du recensement agricole de 2000 (RGoB 2000), l'information n'ayant pas été recueillie pour les recensements ultérieurs. Les estimations de la population animale datent par contre du recensement de 2008 (RGoB 2008) 
Photo 11. Hybrides utilisés pour le transport de céréales des terres de basse d'altitude au village d'éleveurs de yaks de Merak

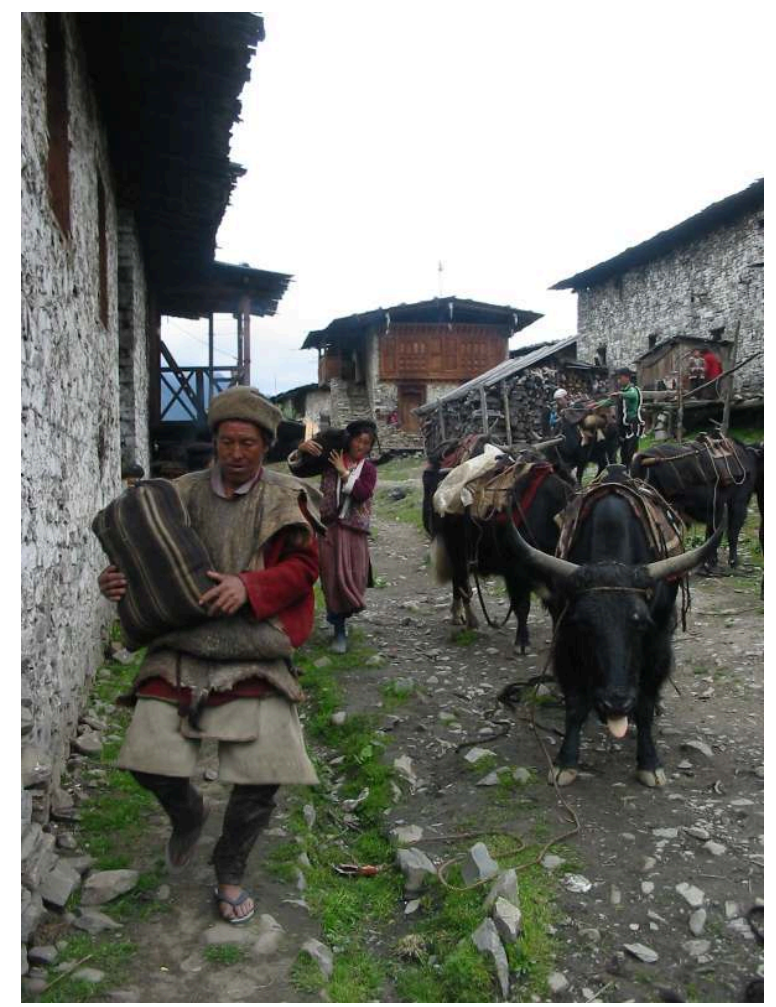

Marie Dervillé (juin 2007, dans le geog de Merak, dans le Dzongkhag de Trashigang)

\section{Revenus et viabilité des systèmes d'élevage : diversités inter- et intra-systèmes}

Les enquêtes réalisées mettent en évidence une grande diversité de structures en termes de taille de cheptel et de revenu au sein des quatre zones identifiées.

À l'exception du système Est, la taille médiane du cheptel est de 50 animaux avec 9 à 11 animaux en lactation. La pratique de l'hybridation explique que les éleveurs de l'est parviennent à subvenir à leur besoin avec des troupeaux de taille plus modeste: médiane à 24 animaux pour 7 animaux en lactation (fig. 8). 
Figure 8. Profil structurel des élevages de yaks dans les différents systèmes régionaux

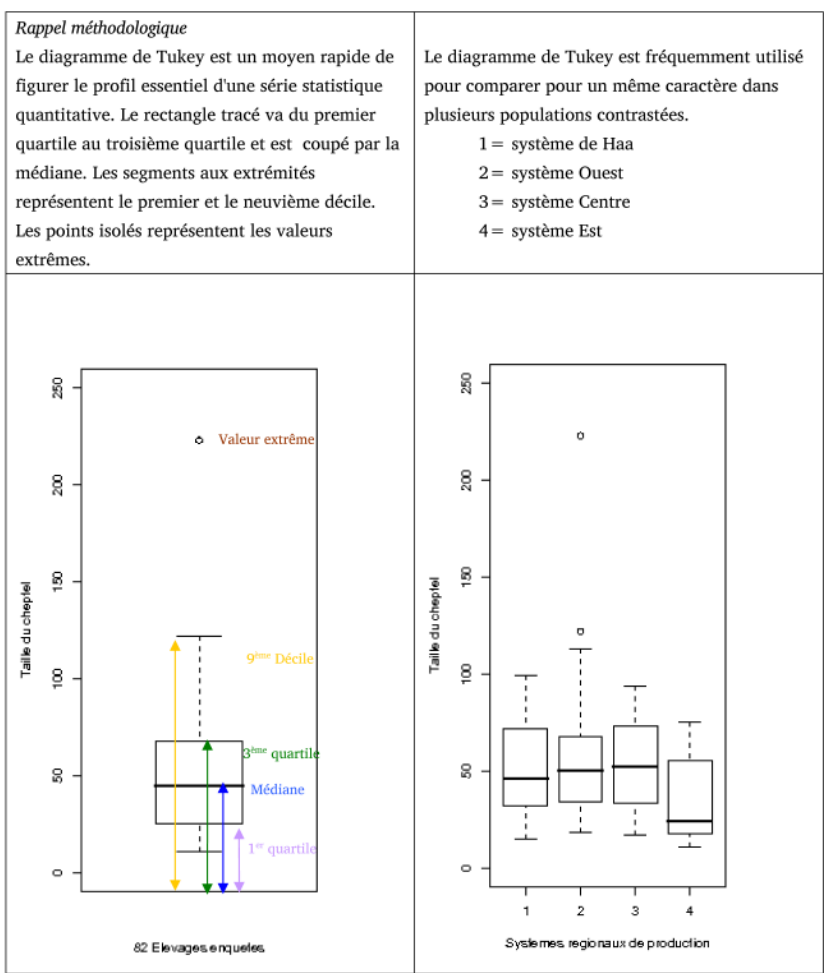

61 Le revenu agricole ${ }^{12}$ annuel moyen des éleveurs de yaks est d'environ $44000 \mathrm{Nu}^{13}$ (977 US\$) ce qui représente une disponibilité mensuelle de $545 \mathrm{Nu}$ (12 US\$) par personne. Le revenu par personne ainsi dégagé est inférieur au seuil de pauvreté estimé pour le Bhoutan par le programme de développement des Nations-Unies en 2003 à $740 \mathrm{Nu} /$ mois (16 US\$/mois) (UNDP 2003) (fig. 9). Avec une valeur ajoutée brute moyenne de $1165 \mathrm{Nu}$ /actif (26 US\$), la majeure partie des éleveurs de yaks voient leur travail rémunéré presque trois fois moins que le salaire mensuel brut minimum de $3000 \mathrm{Nu}$ (67 US\$). 
Figure 9. Performances économiques de l'élevage de yaks : comparaison de la situation dans les 4 systèmes régionaux

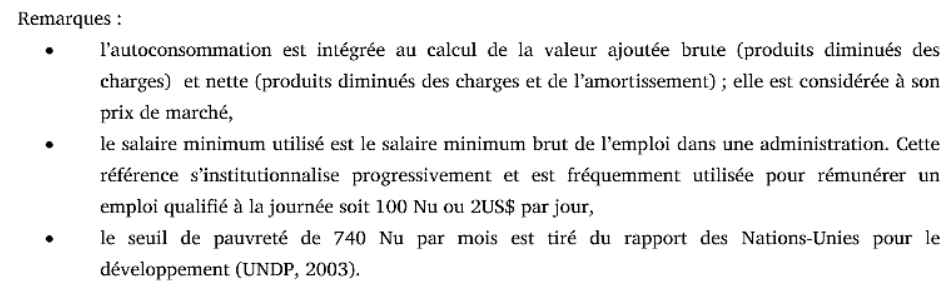

- l'autoconsommation est intégrée au calcul de la valeur ajoutée brute (produits diminués des charges) et nette (produits diminués des charges et de l'amortissement) ; elle est considérée à son prix de marché,

- le salaire minimum utilisé est le salaire minimum brut de l'emploi dans une administration. Cette référence s'institutionnalise progressivement et est fréquemment utilisée pour rémunérer un emploi qualifié à la journée soit $100 \mathrm{Nu}$ ou $2 \mathrm{US} \$$ par jour,

- le seuil de pauvreté de $740 \mathrm{Nu}$ par mois est tiré du rapport des Nations-Unies pour le développement (UNDP, 2003).

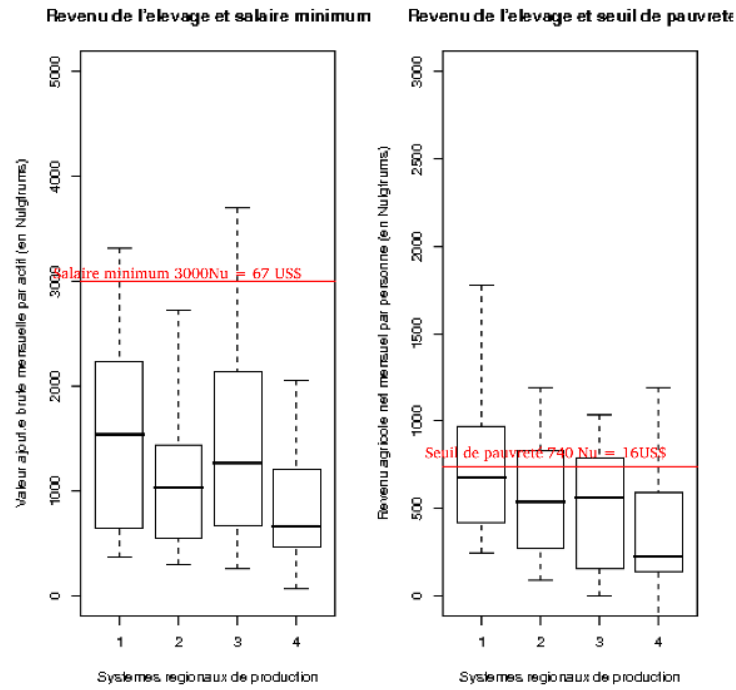

Toutefois, ces chiffres nationaux masquent de grandes disparités inter- et intrasystèmes (fig. 9). Le revenu moyen par personne varie entre 455 (10 US\$/mois, système Est) et $1040 \mathrm{Nu} /$ mois ( 23 US\$/mois, système de Haa). La spécificité du système Est corrobore les résultats de l'étude sur le seuil de pauvreté au Bhoutan : $2 / 3$ des pauvres sont en effet des ruraux avec une concentration marquée dans les districts de l'est du pays. Toutefois, ce propos doit être nuancé car une partie de cet écart de revenu s'explique par un coût de la vie et un coût d'opportunité ${ }^{14}$ de la main d'œuvre moindres ${ }^{15}$. L'écart de revenu en faveur des systèmes de Haa et Centre peut s'expliquer dans le premier cas par la pratique d'une activité viande et, dans le second, par une taille de cheptel en moyenne supérieure ainsi que par un niveau de valorisation des produits laitiers élevé.

La pratique de la vente d'animaux pour l'abattage constitue un élément fort de la diversité inter-systèmes des revenus. Elle est courante dans les systèmes Ouest et de Haa où elle représente respectivement $50 \%$ et $60 \%$ du produit brut de l'élevage de yaks. Elle est plus occasionnelle dans les systèmes Centre et Est où elle constitue moins de $12 \%$ du produit brut. Un mâle en bonne condition se négociant en moyenne 20-25 $000 \mathrm{Nu}$ en 2009 (soit 444-555 US\$), la production de viande constitue donc une part non négligeable du revenu.

Deux facteurs peuvent expliquer la faiblesse relative de la valeur ajoutée brute par actif dans le système Ouest : un nombre d'actifs relativement important, en lien avec une pression relativement faible sur la main d'œuvre et un poids important du métayage ; en effet, $45 \%$ des éleveurs rencontrés sont en métayage ou assimilés. Cette pratique est également fréquente dans le système Centre ( $35 \%$ des éleveurs) mais avec un impact sur le revenu plus modéré. Dans ces deux systèmes, le prélèvement des monastères ou 
des grands propriétaires est loin d'être négligeable. Il représente plus de la moitié de la production de beurre des éleveurs concernés : $83 \mathrm{~kg}$ de beurre soit $19090 \mathrm{Nu}$ (424 US\$, équivalent calculé au prix moyen de la zone) dans le système Ouest et $46 \mathrm{~kg}$ soit 11960 $\mathrm{Nu}$ (266 US\$) dans le système Centre, auxquels s'ajoutent la viande et le pelage en cas de perte ou d'abattage.

Enfin, la taille des troupeaux, l'efficacité et la capacité à valoriser les produits jouent vraisemblablement sur le niveau de revenu des éleveurs et contribuent à expliquer la variabilité intra-système.

Par ailleurs, l'articulation avec d'autres activités joue sur la viabilité des systèmes. Ainsi, dans les systèmes Centre et Ouest, les éleveurs de yaks disposent, avec la cueillette de plantes et champignons médicinaux, de revenus alternatifs conséquents. Le revenu généré en un mois par la cueillette du cordyceps est en moyenne de 600 US\$ et de 900 US\$ respectivement dans ces systèmes. Ce revenu est proche du revenu généré par l'activité d'élevage en un an (1000 US\$). Il est 30 à 45 fois supérieur à la rémunération mensuelle de la main d'œuvre par l'activité d'élevage ( 20 US\$).

Le commerce transfrontalier constitue également une source de revenu non négligeable (1000 US\$ en moyenne pour les éleveurs qui le pratiquent et jusqu'à 3000 US\$ par an dans certains cas). Toutefois, contrairement à la cueillette des cordyceps, la pratique de cette activité requiert un capital initial, sous forme de chevaux pour assurer le transport et de trésorerie pour acheter les biens de consommation; elle n'est donc pas accessible à tous les éleveurs. En outre, il semble que les opportunités varient d'une région à l'autre ; elles seraient supérieures dans les systèmes de Haa et Est.

Les systèmes de Haa et Centre se distinguent quant à eux par le fait que la famille élargie possède des terres agricoles de basse altitude (1 500-2 $500 \mathrm{~m}$ ). Les échanges de produits (céréales, radis et vêtements contre beurre, viande et fromage) et de services (scolarisation des enfants) sont nombreux.

Donc, tourisme, commerce, échange de fonds au sein de la famille et, surtout, cueillette du cordyceps, constituent des compléments de revenu non négligeables. Lorsque l'on considère la totalité des revenus, la médiane se situe autour du seuil de pauvreté, à l'exception du système Est ${ }^{16}$ (fig. 10). Dans le système Ouest, l'impact des revenus générés par la cueillette du cordyceps est particulièrement fort : cette activité permet à la majorité des familles d'éleveurs de vivre au dessus du seuil de pauvreté. 
Cette estimation du revenu total des éleveurs a été réalisée à partir de la valeur ajoutée nette à laquelle ont été ajoutés les revenus issus de l'élevage ovin, du commerce, du portage, de la cueillette de plantes médicinales ou encore les transferts monétaires ou en nature au sein de la famille élargie (source : enquêtes 2007)

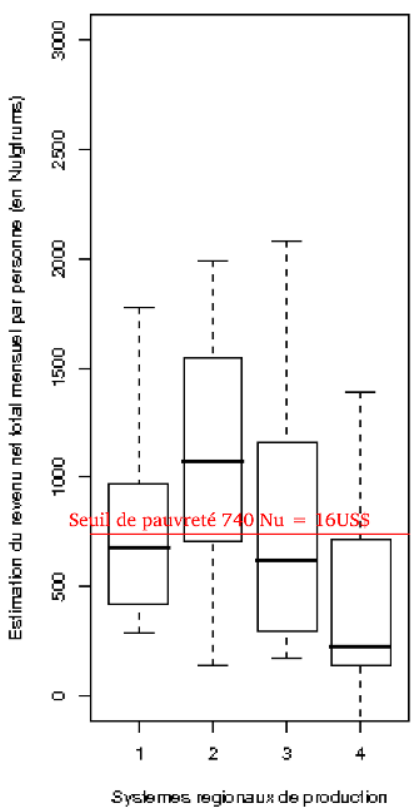
subsistance d'une famille. Toutefois, la viabilité de l'activité ne semble pas suffire à la rendre attractive. Les récits de cessation sont plus nombreux que ceux d'installation: l'activité semble sur le déclin.

\section{Une activité en déclin plus ou moins rapide selon les régions}

D'après les recensements agricoles, la population de yaks aurait cru de 32911 en 1986 à 48400 en 2008. La hausse semble avoir été particulièrement marquée entre 2000 et 2005 (+37\%) et plus particulièrement à l'est du pays (+61\%). En l'absence d'importations, cette hausse des effectifs est surprenante. Elle est, en outre, en contradiction avec la perception des acteurs de terrain. Le taux de mortalité aurait pu être particulièrement élevé en 2000 du fait d'aléas climatiques mais les enquêtes n'ont rien mis de tel en évidence. Un programme d'éradication de la cœnurose a bien été mené, mais dans les années 1970. La radicalisation du bouddhisme ${ }^{17}$ et la stigmatisation croissante de l'abattage ${ }^{18}$ a pu jouer sur le nombre d'animaux abattus d'autant que, du fait de la modernisation de l'économie, de nouvelles sources de revenus (plantes médicinales, commerce, transfert de fonds au sein de la famille élargie, tourisme) se sont développées, réduisant, pour certains éleveurs, le besoin financier de la vente d'animaux pour la production de viande.

Il est aussi probable, en l'absence de système d'identification des yaks, qu'une partie des variations entre 2000 et 2005 s'expliquent par une différence de méthode de recensement ${ }^{19}$. Depuis 2005 la population de yaks semble s'être stabilisée, les hausses d'effectifs (système Ouest notamment) compensant les baisses (système de Haa 
surtout). Si les chiffres sont à considérer avec un œil critique, ils suggèrent en tout cas une différenciation des dynamiques régionales (fig. 11).

Figure 11. Évolution de la population de yaks et hybrides

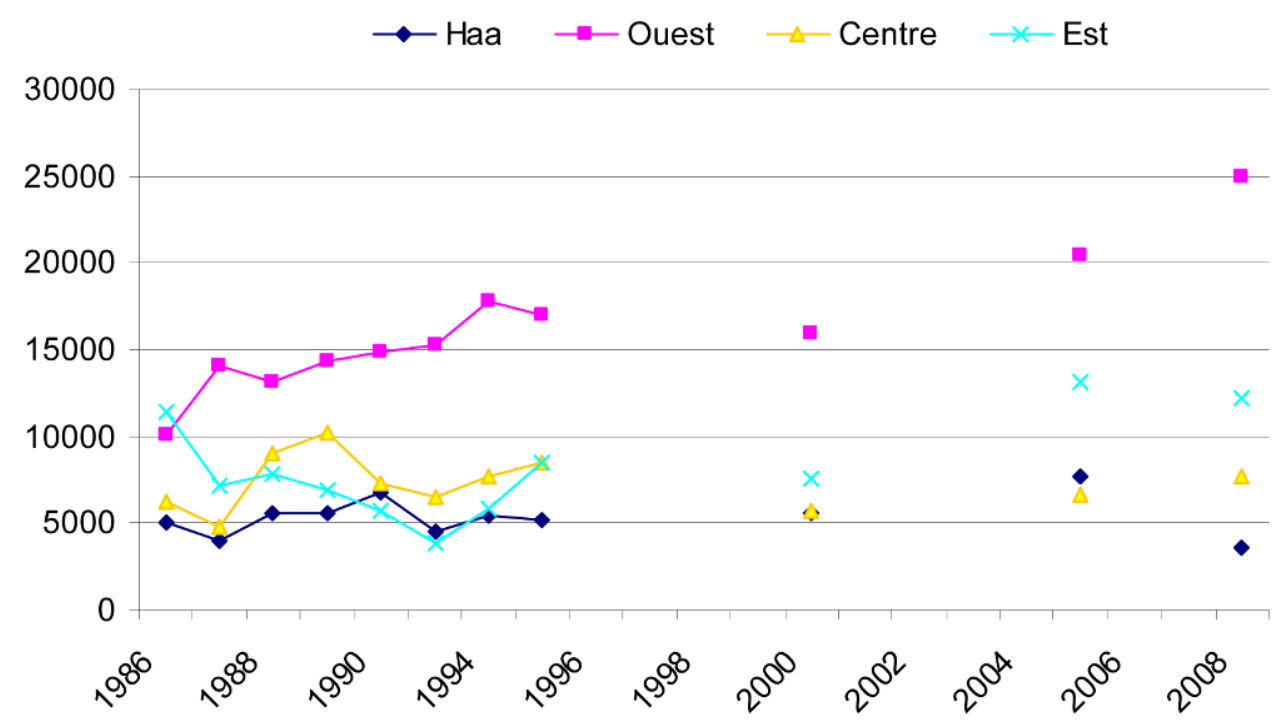

Source: Recensement agricole (RGoB)

Dans les zones où l'élevage de yaks est une activité secondaire de la famille élargie (Bumthang, Haa), le déclin de l'activité est avéré. Alors que l'activité était pratiquée dans trois des quatre geogs de la région de Bumthang, elle ne l'est plus que dans un seul (Chokorte). À Haa, dans les trois geogs où l'activité est pratiquée, $60 \%$ des familles détenaient un troupeau il y a encore dix ans ; elles ne sont plus que $10 \%$ aujourd'hui soit 51 familles $^{20}$. Ces résultats suggèrent que, plus que le niveau de rémunération du travail, c'est le coût d'opportunité de la main d'œuvre qui est le principal déterminant de la cessation d'activité : lorsque de nouvelles opportunités d'emplois s'ouvrent, il croît et les incitations à cesser l'activité augmentent.

Dans les zones où l'élevage de yaks reste l'activité principale, la pression sur l'activité est également forte. Si des investissements sont encore réalisés dans l'activité - achat de yaks des régions en déprise -, ils sont concomitants d'investissements extérieurs à l'activité (éducation des enfants, achat de terres agricoles, achat de fonds de commerce). Dans le geog de Sephu dans la région de Wangdue, le nombre de familles d'éleveurs a diminué de $18 \%$, passant de 62 à 51 entre 2000 et 2009.

$\mathrm{Si}$ les évolutions restent contrastées entre régions, les récits d'éleveurs et de techniciens d'élevage renvoient plutôt l'image d'une activité sur le déclin. Ce déclin est beaucoup plus rapide pour les systèmes d'élevage les plus intégrés à la société moderne $\mathrm{du}$ fait de la possession, dans le cadre de la famille élargie, de terres agricoles de basse altitude qui ouvrent l'accès aux services publics et aux opportunités économiques.

Compte tenu des observations réalisées en 2009 dans les régions de Haa et de Wangdue, il est probable que le nombre de foyers impliqués dans l'activité d'élevage de yaks ait diminué par rapport aux 1400 foyers recensés en 2000. Si on considère une baisse de population intermédiaire de $20 \%$ comme à Wangdue, le nombre de familles d'éleveurs se chiffre en 2008 probablement aux alentours de 1100. 


\section{Conclusion : Des systèmes d'élevage en situation de grande fragilité}

77 Ce travail a permis de dresser un tableau des pratiques des éleveurs de yaks bhoutanais, de la conduite du troupeau à la commercialisation des produits. L'élevage apparaît comme une activité familiale de subsistance imbriquée dans des relations sociales complexes. La productivité limitée s'explique pour partie par un accès limité aux intrants et à l'appui technique. Du fait de l'éloignement des axes routiers, l'élevage de yaks et la transformation des produits issus de cet élevage sont en effet restés des activités manuelles, intensives en travail mais extensives en capital.

La différenciation régionale des pratiques et des environnements socio-économiques conduit à distinguer quatre systèmes régionaux d'élevage.

L'analyse économique des revenus des éleveurs qui a pu être menée dans ces différents systèmes met en évidence que les éleveurs de yaks, avec un revenu annuel moyen proche de 1000 US\$, rémunèrent la main d'œuvre familiale environ au tiers du salaire brut minimum bhoutanais et, dans nombre de cas, ne permettent pas à leur famille de vivre au dessus du seuil de pauvreté tel qu'il a été défini par l'UNDP en 2003. Toutefois, on note une grande variabilité intra- et inter-systèmes: une taille plus grande des troupeaux, l'accession à un statut privé du cheptel et surtout la vente régulière d'animaux pour la production de viande semblent des éléments déterminants du niveau de revenu dégagé par l'activité. Lorsque la totalité des revenus des éleveurs (incluant les revenus tirés du commerce, du tourisme et surtout de la cueillette du cordyceps) est considérée, une large proportion des éleveurs se hisse au dessus du seuil de pauvreté. Ainsi l'élevage de yaks, en articulation avec d'autres activités connexes agricoles ou non, peut encore permettre d'assurer la subsistance d'une famille. Toutefois il n'en reste pas moins sur le déclin, avec une diminution du nombre de familles impliquées dans l'activité de l'ordre de $20 \%$ au cours de ces huit dernières années. La différenciation régionale des dynamiques s'explique vraisemblablement par un coût d'opportunité contrasté de la main d'œuvre familiale. Le coût d'opportunité de l'activité est le plus fort, et le déclin de l'activité le plus marqué, lorsque les alternatives à l'activité sont nombreuses. Les systèmes Haa et Centre plus directement intégrés à la société modernisée par le biais de la famille élargie sont directement concernés. Compte tenu des écarts de revenu, entre élevage et activités annexes (commerce et cueillette de plantes médicinales), il est probable qu'une concurrence entre ces activités dans l'emploi de la main d'œuvre se fait de plus en plus jour - concurrence qui risque de déstabiliser les élevages les plus éloignés des axes de communication, comme le système Ouest.

Le lien entre fragilité du système d'élevage et coût d'opportunité de la main d'œuvre suggère que l'insertion de l'activité dans la société est devenue problématique. Ce constat invite à changer d'échelle et à inclure d'autres acteurs dans le champ de l'analyse: acteur public, acteurs du tourisme, consommateurs urbains, etc. Avec la modernisation de l'économie bhoutanaise, il est en effet probable que les règles à l'origine de l'élaboration de la valeur des ressources et des produits de l'activité d'élevage et donc, à l'origine de sa rentabilité, ne soient plus uniquement locales. J'analyserai dans l'article suivant comment l'évolution des réglementations nationales et la monétisation de l'économie contribuent à la déstabilisation des systèmes d'élevage (Dervillé 2012). 


\section{BIBLIOGRAPHIE}

Bonnemaire, J.

2001 Enjeux sur les savoirs et les objets de la zootechnie : L'élevage entre science, technologie, nature et société, Comptes rendus de l'Académie d'agriculture de France 87(4), pp. 237-260.

Bonnemaire, J., Jest, C., Osty, P. L. \& Verrier, E.

2004 Mountain livestock farming and sustainable development : insights from the French experience (Chengdu, Sichuan, Fourth international Congress on Yak), $15 \mathrm{p}$.

CNIEL

2009 Economie laitière en chiffres, 180 p.

Department of Livestock

2008 Livestock statistics 2008 (Thimphu, Ministry of Agriculture), 155 p.

Dervillé, $\mathrm{M}$.

2012 L'élevage des yaks au Bhoutan : II. Un symbole menacé de marginalisation par la modernisation de l'économie. Quelles politiques publiques pour préserver ce patrimoine, EMSCAT 43 (à paraître).

Dervillé, M. \& Bonnemaire, J.

2010 Marginalisation of yak herders in Bhutan : can public policy support the transformation of their skills and organisations? (Montpellier, ISDA), $10 \mathrm{p}$.

Dong, S. K., Long, R. J. \& Kang, M. Y.

2003 Milking and milk processing : traditional technologies in the yak farming system of the Qinghai-Tibetan plateau, China, International Journal of Dairy Technology 56(2), pp. 86-94.

FAO

2006 Dossiers spéciaux : Situation des disponibilités et de la sécurité alimentaires en Mongolie, in Perspectives de récoltes et situation alimentaire (Rome, FAO / Département économique et social), pp. 26-30.

Karma Ura

1993 The nomad's gamble : pastoralist of northern Bhutan, South Asia Research 13, pp. 81-100. 2002 The herders' dilemma, Journal of Bhutan studies 7, pp. 1-43.

Kusang Choden

2008 Chilli and cheese. Food and society in Bhutan (Bangkok, White Lotus), 227 p.

Landais, E. 1994 Système d'élevage. D'une intuition holiste à une méthode de recherche, le cheminement d'un concept. Dynamique des systèmes agraires, in Blanc-Pamard, C. \& Boutrais, J. (éd.), À la croisée des parcours : pasteurs, éleveurs, cultivateurs (Paris, ORSTOM, Collection colloque et séminaires), pp. 15-49.

1998 Agriculture durable : les fondements d'un nouveau contrat social ?, Le courrier de l'environnement 33, $15 \mathrm{p}$.

Mission Economique de Pékin 2006 Transformation et consommation des produits laitiers en Chine (Pékin, MINEFI / DGPTE), $4 \mathrm{p}$. 
Pema Gyamthso

1996 Assessment of the condition and potential improvment of high altitude rangeland of

Bhutan. (Agronomy. Zurich. PhD Thesis).

Pommaret, F.

2000 Le Royaume du Bhoutan, téléchargé le 21 décembre, 2010.

(http://www.amisdubhoutan.fr/OldSite/Pages/bhoutan.pdf), 9 p.

Roder, W. \& Pema Gyamtsho

2001 Feeding the Herds : Improving fodder resources in Bhutan (Katmandu, ICIMOD), 124 p.

UNDP

2003 Bhutan Living standard survey, Thimphu, Bhutan, 108 p.

Tashi Dorji

2000 Genotypic and phenotypic characterisation of the yak (bos grunniens) and yak farming

systems in Bhutan (Institute of Land and Food resources, University of Melbourne, M. Sc. Thesis).

Wiener, G., Han Jianlin \& Long Ruijun

2003 The Yak second edition (Bangkok, FAO), $460 \mathrm{p}$.

\section{NOTES}

1. Le geog est une unité administrative intermédiaire entre le village et le Dzongkhag.

2. Le terme a été choisi pour rendre compte d'une situation intermédiaire entre transhumance et nomadisme dans la mesure où l'habitation d'hiver est fixe et où les lieux de pacage sont multiples (de 4 à 10 dans les entretiens réalisés).

3. Le recensement de 2008 fait état d'une population de 48400 animaux. Le recensement agricole de 2000 est le seul à relater le nombre de foyers impliqués dans l'activité, estimé à 1400 en 2000 . Compte tenu d'une baisse que l'on peut estimer à $20 \%$ d'après les enquêtes réalisées, le nombre de familles d'éleveurs de yaks fin 2008 se situe probablement aux alentours de 1100 familles.

4. L'hybridation était autrefois pratiquée dans le centre du pays où la force de traction des mâles hybrides était recherchée pour le labour mais, avec l'usage croissant de motoculteurs au sein des exploitations de la région, cette pratique a disparu.

5. La famille est dite élargie lorsqu'oncles et tantes travaillent en indivision sur le patrimoine familial. Chaque couple a toutefois souvent une responsabilité bien définie : agriculture pour l'un, élevage bovin pour un autre, élevage de yaks pour le dernier. La charge d'élever le troupeau de yaks est confiée, à chaque génération, à un des membres de la famille, le plus souvent le fils ou la fille de l'éleveur de la génération précédente. Lorsque les éleveurs de yaks dépendent totalement de l'activité, la famille élargie gère le troupeau en indivision ou choisit de le partager entre les descendants selon les cas. Une étude socio-anthropologique plus approfondie serait nécessaire pour mieux comprendre les déterminants éventuels d'un découplage d'unités de travail au sein de la famille élargie.

6. Pour réaliser cette estimation, les consommations de lait et de beurre ont été prises en compte. Le fromage étant issu du babeurre, il n'a pas été pris en compte dans les calculs. Un kilogramme de beurre, tel que mis en évidence par le travail d'enquête, a été évalué à $16 \mathrm{~kg}$ équivalent lait. Il s'agit d'une estimation déduite de la différence entre quantités produites et quantités commercialisées collectées lors des enquêtes réalisées en 2007.

7. Dr Pema Gyamtsho, comme mentionné plus haut, est l'auteur d'une thèse sur les potentiels et les conditions d'amélioration des pâturages d'altitude (1996). Il est aussi depuis 2008 Ministre de l'Agriculture du Bhoutan. 
8. skyesmed chimed est l'écriture phonétique d'une expression dzongkha qui signifie "pas de naissance pas de mort ".

9. La cœnurose est une maladie parasitaire. La larve du ver se développe dans le cerveau des bovins, équins, ovins et caprins leur faisant perdre l'équilibre et cesser de s'alimenter. Il n'existe pas de traitement curatif. Le contrôle de la population de chiens (hôtes du stade final du parasite) et l'enterrement des carcasses des animaux atteints constituent le principal traitement préventif. 10. Les chiffres sont issus des recensements agricoles 2000 pour le nombre de foyers et 2008 pour la population de yaks.

11. Historiquement, le rôle de commerçant ou de messager des éleveurs de yaks était institutionnalisé. Il a ensuite été interdit suite à l'annexion du Tibet par la Chine et à la fermeture de la frontière. Depuis une dizaine d'années, des échanges commerciaux informels sont tolérés par les gardes frontières : quelques produits issus de l'élevage de yaks sont échangés mais il s'agit surtout d'importation de couvertures, de tasses, de thermos, de chaussures et autres produits manufacturés à destination des marchés urbains.

12. Le revenu agricole est la différence entre le produit brut d'une exploitation agricole et l'ensemble des charges fixes et variables pour une période donnée. Il prend en compte l'autoconsommation des éleveurs qui est intégrée au produit. L'autoconsommation est prise en compte à sa valeur de marché. Par ailleurs, seules l'autoconsommation et la vente de produits laitiers et de viande ont été prises en compte dans le calcul du revenu. Le fait que le filage et le tissage soient en perte de vitesse, ainsi que l'utilisation des yaks comme animaux de travail, justifie ce choix. Dans certains cas, notamment pour effectuer une comparaison avec le salaire minimum brut, on raisonnera en termes de valeur ajoutée brute qui représente la différence entre produits et charges variables.

13. Le Nulgtrum $(\mathrm{Nu})$ est la monnaie bhoutanaise. Le taux de change utilisé pour effectuer les conversions en dollars est le taux de change moyen de l'année 2007 soit $45 \mathrm{Nu}$ pour 1 US\$.

14. Le coût d'opportunité est un coût de renoncement lié au fait qu'un facteur de production, ici la main d'œuvre, n'est plus disponible pour une autre production du fait de son engagement dans une activité qui pourrait la rémunérer à ce coût, ici l'élevage de yaks. Le coût d'opportunité d'un facteur de production est en général estimé par la rémunération de ce facteur de production dans son emploi le meilleur sur la zone : dans le cas présent, pour le facteur main d'œuvre, c'est le salaire des journaliers agricoles et des artisans ruraux qui définit le coût d'opportunité de la main d'œuvre.

15. A titre d'exemple, le prix d' $1 \mathrm{~kg}$ de beurre de yak varie avec la zone géographique - en moyenne $130 \mathrm{Nu}(2,8 \mathrm{US} \$ / \mathrm{kg})$ à l'est du pays, $200 \mathrm{Nu}(4,4 \mathrm{US} \$ / \mathrm{kg})$ à Haa, $230 \mathrm{Nu}$ à l'ouest et $260 \mathrm{Nu}(2,8 \mathrm{US} \$ / \mathrm{kg})$ à Bumthang ; de même que le salaire d'un journalier : $80 \mathrm{Nu}$ (1,7 US\$) à l'est, $100 \mathrm{Nu}(2,2$ US\$) à l'ouest, $120 \mathrm{Nu} /$ jour (2,6 US\$) à Haa et Bumthang.

16. Les revenus sont bien sûr difficiles à estimer notamment parce que la pratique du commerce transfrontalier, activité illicite juste tolérée ces dernières années, n'a pas été mentionnée systématiquement par les éleveurs. Les revenus du commerce seraient de fait, du point de vue des techniciens d'élevage rencontrés, plutôt sous-estimés.

17. La radicalisation du Bouddhisme n'est pas propre au Bhoutan mais se retrouve aussi dans les communautés tibétaines en exil en Inde et au Népal qui se rapprochent d'un régime favorisant le végétarianisme. Ceci est en partie à attribuer à l'influence des Occidentaux bouddhistes et des Indiens. (Pommaret, communication personnelle 2009).

18. Le Je Khempo, l'autorité religieuse du pays, défend l'idée que les Bhoutanais doivent réduire leur consommation de viande. Trois niveaux de stigmatisation ont été identifiés lors des entretiens : l'abattage, l'investissement dans une activité de production de viande et enfin la consommation de viande. Elle n'est tolérée que dans le cas de viande issue d'animaux morts de causes naturelles. Le tabou varie aussi avec le type de viande. Les bouddhistes pratiquant sont invités à cesser la consommation de poisson, de porc et d'œufs « Nga, Pha Gongsum » (Kunzang 
Choden 2008). Le végétarianisme se développe principalement en ville mais les moines exercent aussi une pression sur les communautés rurales, prêchant contre la consommation de viande à l'occasion des nombreuses cérémonies religieuses de la vie bhoutanaise. L'élevage de porc d'autoconsommation, traditionnellement pratiqué par toute famille rurale comme valorisation des déchets domestiques, est notamment sur le déclin (Kunzang Choden 2008). L'accroissement des importations bon marché et l'achat occasionnel de viande s'y substituent. Le département d'élevage a tenté plusieurs fois ces trente dernières années de développer des activités ayant trait à la production de viande mais, à chaque fois, les projets ont dû être abandonnés sous la pression de l'autorité religieuse et d'une partie de la population. La majorité des abattoirs du pays ont été fermés.

19. Le recensement agricole de 2000 était un recensement de grande ampleur conduit sous l'égide du ministère de l'agriculture. Le questionnaire était national, chaque foyer a été visité. Le taux de couverture a été estimé à $87 \%$. Le recensement de 2005 et les suivants ont été décentralisés et confiés aux départements d'agriculture et d'élevage. Pour l'élevage, la responsabilité de fournir des données actualisées a été transférée aux techniciens d'élevage. La collecte de données a perdu de son caractère formel, les techniciens ne visitant pas systématiquement tous les élevages.

20. Ces chiffres sont issus d'enquêtes complémentaires que j'ai réalisées en 2009, notamment auprès des élus de ces deux geogs.

\section{RÉSUMÉS}

Cet article analyse la viabilité des systèmes d'élevage de yaks au Bhoutan au travers d'une approche systémique. La différenciation régionale des pratiques associées à cet élevage seminomade a permis l'identification et la caractérisation de quatre systèmes régionaux d'élevage. L'évaluation des performances technico-économiques de l'activité révèle une grande variabilité inter- et intra- système. La taille et le statut du cheptel, ainsi que la vente régulière d'animaux permettent d'accroître les revenus. Toutefois, dans la majorité de cas, l'élevage des yaks ne parvient pas à rémunérer le travail familial au salaire minimum. Le déclin de l'activité observé sur le terrain peut ainsi être expliqué.

This article examines the viability of yak husbandry in Bhutan through a systemic approach. Regional differences in this semi-nomadic form of livestock rearing suggest four distinct systems. Technical-economic performances vary greatly among and within these systems. The size and the status of the herd as well as the regular sale of animals lead to increased income. Yet it appears that yak farming remunerates family labour below the minimum wage. The decline of the activity observed in the field can thus be explained.

\section{INDEX}

nomsmotscles Bhoutanais

Index géographique : Bhoutan, Himalaya

Mots-clés : yak, élevage, nomadisme, économie

Keywords : yak, herding, nomadism, economics 


\section{AUTEUR}

\section{MARIE DERVILLÉ}

Marie Dervillé est agroéconomiste. Elle a réalisé un travail de recherche-action de près de trois ans au Bhoutan. Elle a effectué diverses missions d'expertise, auprès du département d'élevage du Ministère de l'Agriculture bhoutanais, dans le cadre d'un programme de coopération avec la France (2005-2007) et d'un programme d'appui au développement de filières agricoles conduit par la FAO (2007 et 2009). Les travaux de l'auteur sur l'élevage laitier en France et en Europe l'ont conduite à la réalisation d'une thèse en Economie Politique sur les capacités d'adaptation de l'élevage laitier de montagne à la libéralisation des marchés européens (2012 Territorialisation du secteur laitier et régimes de concurrence : le cas des montagnes françaises et de leur adaptation à l'après-quota). 